\title{
AMENAZA Y RIESGO SÍSMICO DEL CONO SUR
}

Omar-Darío Cardona ${ }^{1, *}$, Gabriel A. Bernal ${ }^{2}$, Daniela Zuloaga ${ }^{2}$,

Mario A. Salgado-Gálvez ${ }^{3}$ y Diana González ${ }^{2}$

\section{RESUMEN}

Se presenta la evaluación del riesgo sísmico en Chile y Argentina, siguiendo un enfoque probabilista, con el objetivo de determinar el perfil de riesgo catastrófico de ambos países. Un modelo de amenaza regional fue definido con base en la caracterización del proceso de subducción, los sistemas de fallamiento intraplaca localizados, un catálogo sismológico actualizado y funciones de atenuación espectrales adecuadas, resultando en curvas, mapas y una colección de eventos generados estocásticamente. La exposición se modeló a partir de una representación aproximada de los activos expuestos (proxi), con base en indicadores socioeconómicos nacionales, obteniendo valores de reposición de construcciones e infraestructura, y para los principales sectores económicos de los países. La vulnerabilidad se definió a partir de funciones de vulnerabilidad apropiadas para cada tipo de infraestructura. El riesgo se calculó de manea totalmente probabilista, en términos de la curva de excedencia de pérdidas y la distribución geográfica de la pérdida anual esperada, para diversos portafolios y de manera separada para Chile y Argentina. Finalmente, se presenta una evaluación detallada del riesgo sísmico de la ciudad de Mendoza, donde se incluye la microzonificación sísmica de la ciudad y el catastro detallado, obteniendo una evaluación a nivel de edificaciones para toda la ciudad.

\section{PALABRAS CLAVE}

Amenaza, riesgo, vulnerabilidad, evaluación del riesgo sísmico

\section{ABSTRACT}

With the objective of determining the catastrophic risk profile of Chile and Argentina, a seismic risk assessment following a probabilistic approach was conducted. A regional hazard model was defined based on the characterization of the subduction process, the localized intraplate fault systems, an up-to-date seismological catalog and appropriate spectral attenuation functions; this resulted in curves, maps and a collection of stochastically generated events. The exposure was modeled using an approximate representation of exposed assets (proxi) based on national socioeconomic indicators, which allowed us to obtain replacement values for buildings and infrastructure, as well as for the main economic sectors of the countries. Asset vulnerability was defined based on vulnerability functions appropriate to each type of infrastructure. The risk was calculated in a totally probabilistic manner in terms of the loss exceedance curve and the geographical distribution of the average annual loss, both for Chile and Argentina individually and for various portfolios in each case. Finally, a detailed evaluation of the seismic risk of the city of Mendoza, including the seismic microzonation of the city and the detailed cadastre, is presented, which resulted in an evaluation with a building-by-building resolution for the whole city.

\section{KEYWORDS}

Seismic hazard, risk, vulnerability, seismic risk assessment
1. Instituto de Estudios Ambientales (IDEA), Universidad Nacional de Colombia Sede Manizales, Manizales, Colombia.

2. INGENIAR Ltda., Bogotá D.C., Colombia.

3. Centre Internacional de Metodes Numerics en Enginyeria (CIMNE), Barcelona, Spain.

*Autor de correspondencia: odcardonaa@unal.edu.co

\section{RECIBIDO}

31 de enero de 2017

\section{ACEPTADO}

20 de febrero de 2017

\section{PUBLICADO}

15 de julio de 2017

Formato cita Recomendada (APA): Cardona, O.D., Bernal, A., G., Zuloaga, D., SalgadoGálvez, M. A. \& González, D. (2017). Amenaza y riesgo sísmico del Cono Sur. REDER, 1(1), pp.43-69.

\section{$(1) \Theta$}

Todos los artículos publicados en REDER siguen una política de Acceso Abierto y se respaldan en una Licencia CreativeCommons Atribución-NoComercial 4.0 Internacional.

Revista de Estudios

Latinoamericanos sobre Reducción del Riesgo de Desastres (REDER) 


\section{INTRODUCCIÓN}

Una de las actividades fundamentales de la gestión del riesgo de desastres a nivel de país es la evaluación del riesgo catastrófico, para lo cual es necesario aplicar metodologías confiables que permitan una adecuada estimación y cuantificación del potencial de pérdidas en un tiempo de exposición determinado. No obstante, aunque se han desarrollado a nivel internacional diversas metodologías para la evaluación detallada del riesgo para diferentes tipos de amenazas naturales, pocas metodologías permiten realizar un análisis a nivel país por dos razones principales: primero, la falta de información detallada que impide la conformación de bases de datos robustas para describir la exposición y, segundo, la falta de metodologías que permitan modelar de manera integrada las amenazas, la vulnerabilidad de los elementos expuestos y el riesgo que se deriva de su respectiva convolución.

Para alcanzar, entonces, el objetivo general de identificar y cuantificar el riesgo catastrófico de un país, es necesario utilizar un método que permita tener en cuenta las amenazas naturales en forma integral, que incluya de la manera más completa, y en lo posible detallada, la exposición de los bienes de infraestructura -teniendo en cuenta sus principales características-, que permita tener en cuenta la vulnerabilidad específica de cada componente de dicha infraestructura y que finalmente permita la evaluación del riesgo mediante un proceso de cálculo probabilista apropiado que tenga en cuenta las incertidumbres propias de un proceso de este tipo, las inevitables limitaciones en la información y la capacidad cómputo electrónico.

En definitiva, la evaluación probabilista del riesgo sísmico tiene como objetivo estimar la distribución de probabilidad de la pérdida que puede presentarse en un conjunto de elementos expuestos, tras la ocurrencia de un terremoto. Es claro que esta evaluación no puede hacerse siguiendo metodologías estadísticas convencionales dado que la frecuencia de ocurrencia de los terremotos es muy baja, por lo cual no se cuenta con registros históricos de pérdida suficientes. La modelación probabilista permite, entonces, realizar pronósticos sobre los niveles futuros de pérdida, considerando la amenaza propia de la región de estudio y la incertidumbre en su estimación, así como la vulnerabilidad inherente de los elementos expuestos y su incertidumbre.

Este trabajo, que tiene como objetivo obtener los perfiles de riesgo catastrófico de Argentina y Chile, presenta la modelación probabilista del riesgo sísmico, la cual incorpora los elementos mencionados anteriormente para derivar métricas de riesgo probabilistas que dan cuenta del potencial de pérdidas asociadas a la ocurrencia de los terremotos. Esta evaluación a nivel nacional pretende brindar información a los tomadores de decisiones de orden nacional con respecto al riesgo por desastres sísmicos. Así mismo, dentro de un marco territorial más acotado, se presenta el caso de estudio del riesgo sísmico en la ciudad de Mendoza, Argentina, calculado usando la misma metodología y obteniendo las mismas métricas, las cuales caracterizan el proceso de ocurrencia de pérdidas por terremotos a nivel local, con el fin de brindar herramientas para la toma de decisiones en el ámbito municipal. Este trabajo ha sido realizado para los dos países en el marco de dos proyectos independientes financiados por el Banco Interamericano de Desarrollo (BID) con objetivo de desarrollar los perfiles de riesgo catastrófico de cada país.

\section{AMENAZA SÍSMICA}

\section{Sismotectónica del Cono Sur}

Por su ubicación en el continente suramericano, la sismo-tectónica de los países de Chile y Argentina es controlada en gran parte por la convergencia entre las Placas de Nazca y Suramérica. Es bien sabido que la Placa de Nazca se desplaza con dirección Este con una velocidad aproximada

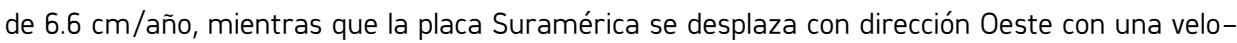
cidad aproximada de $3 \mathrm{~cm} /$ año - traduciéndose en una tasa neta entre $8-9 \mathrm{~cm} /$ año (Kendrick et al., 2003) - , generando una extensa zona de colisión entre ambas placas. A lo largo de esta convergencia la Placa oceánica de Nazca (más densa) se introduce en el manto terrestre bajo la Placa Suramérica en un proceso de subducción, creando una zona de alta sismicidad a lo largo de todo el borde occidental del continente.

Por otro lado, en la parte sur del continente ocurre otro proceso de subducción, aunque a menor escala, esta vez entre las Placas Antártica y Suramérica. La Dorsal de Chile es la estructura submarina que genera y separa las placas de Nazca y Antártica a una latitud aproximada de $46^{\circ} \mathrm{S}$, punto a partir del cual la Placa Antártica subduce bajo Suramérica. No obstante, la velocidad de convergencia entre estas dos placas es relativamente menor y por esta razón la actividad sísmica 
es más baja. Finalmente, y más hacia el sur del continente, la Placa de Scotia se encuentra al sur de la Placa Suramericana, en la punta del continente, y se mueve lateralmente con respecto a la Placa Antártica y a la suramericana. En este caso no hay subducción y el movimiento entre placas es de menor magnitud, por lo que la actividad sísmica es aún más reducida.

Los diferentes movimientos entre las placas tectónicas mencionadas crean, además, esfuerzos dentro de la placa continental, generando así fallas geológicas al interior de la misma. Estas fallas, que en general son de carácter superficial, son fuentes adicionales de sismicidad que pueden afectar áreas alejadas de la zona de subducción. Todo lo anterior aporta a una alta sismicidad en esta región. La Figura 1 muestra el entorno tectónico, los lineamientos de las fallas geológicas conocidas y el catálogo sismológico de la región.

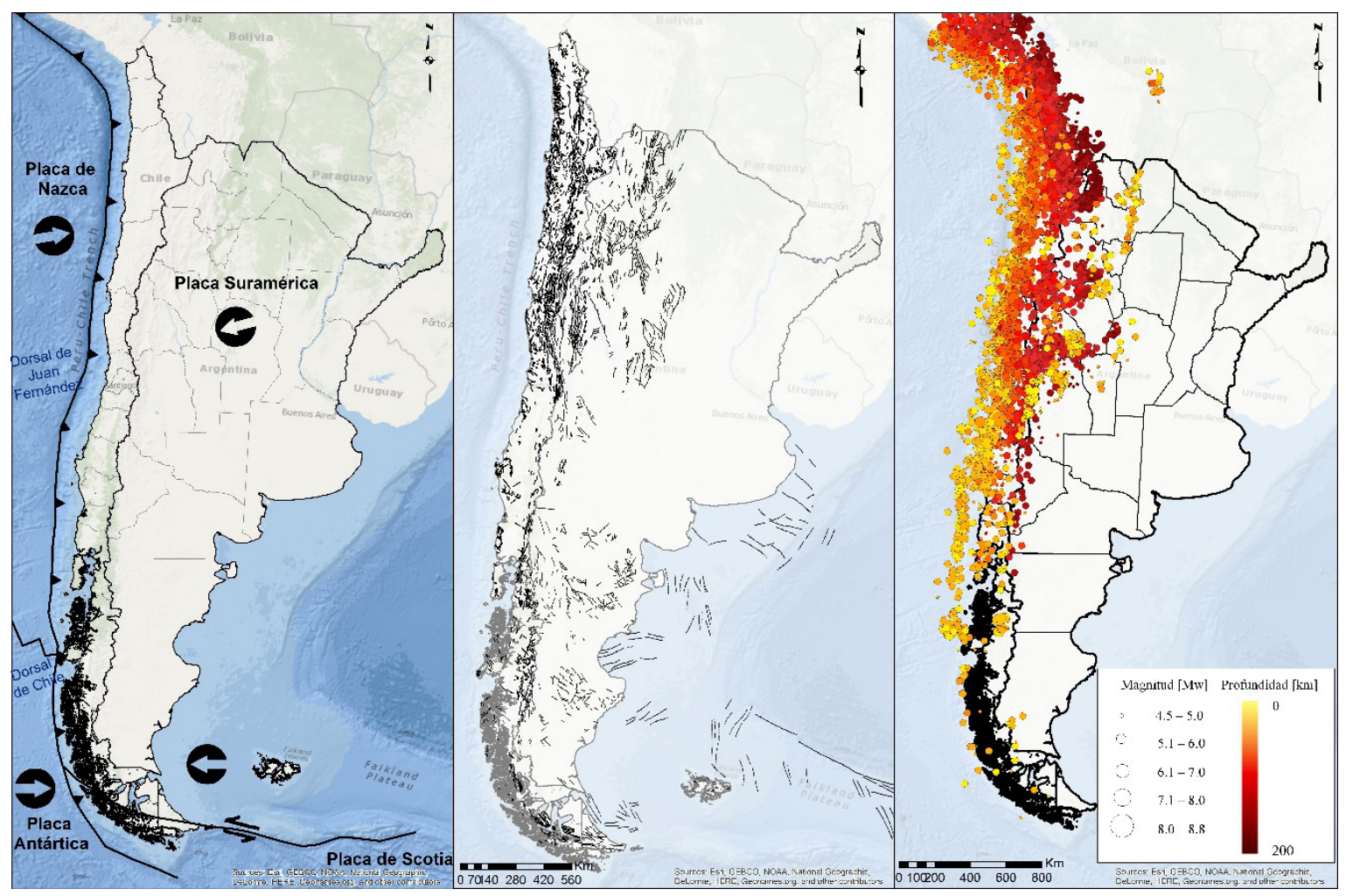

Figura 1. Entorno tectónico (izquierda), fallas geológicas (centro) y catálogo sismológico (derecha). Adaptado de: Costa et al., 2000; Lavenu et al., 2000; SEGEMAR, 2015; SERNAGEOMIN, 2014.

\section{Modelación de la amenaza sísmica}

La amenaza sísmica se modeló siguiendo la metodología de evaluación probabilista PSHA (Esteva 1967, Cornell 1968). Primero, se definieron fuentes sismogénicas representativas de los principales accidentes tectónicos y lineamientos de fallas en la región. Estas fuentes se dividieron en dos tipos: (1) fuentes de subducción, que son fuentes profundas, y (2) fuentes intraplaca, que son fuentes superficiales dentro de la placa suramericana, esto con el fin de diferenciar ambientes sismotectónicos. La Figura 2 muestra todas las fuentes sismogénicas definidas para esta región; a la izquierda se presentan las fuentes intraplaca, mientras que a la derecha se presentan las fuentes de subducción. Todas las fuentes, tanto las intraplaca como las profundas, fueron modeladas como planos inclinados, para así considerar no sólo la geometría de la proyección en superficie, si no los buzamientos y profundidades que cada fuente alcanza.

La definición de estas fuentes sismogénicas se realizó en base a una extensa revisión de la literatura existente, identificando los sistemas más importantes y de reconocimiento científico (Silva, 2008; Costa et al., 2000; Pingel et al., 2013; Masaferro et al., 2003; DeCelles et al., 2011; Carrapa \& DeCelles, 2008; Deeken et al., 2006; Kley \& Monaldi, 1999; laffa et al., 2013; Jordan \& Allmendinger, 1986; Ramos et al., 2001; Fazzito, 2011; SEREMI, 2011; Vargas et al., 2012; Costa, 2004; Perucca \& Vargas, 2014; Salomon et al., 2013; Leyton et al., 2010; SERNAGEOMIN, 2014 Núñez, 2014; Armijo et al., 2010; CSN, s.f). 


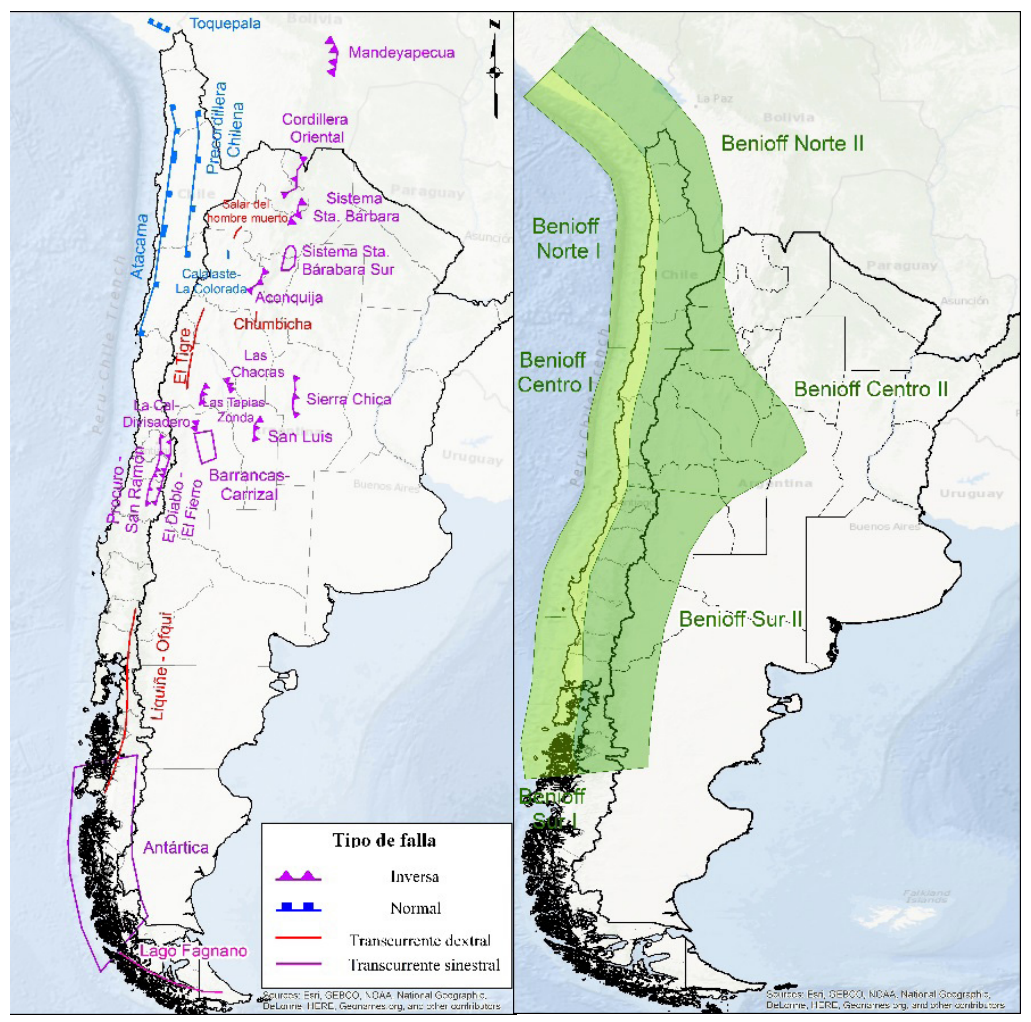

Figura 2. Fuentes sismogénicas superficiales (izquierda) y profundas (derecha). Adaptado de: Costa et al., 2000; Lavenu et al., 2000; SEGEMAR, 2015; SERNAGEOMIN, 2014

Enseguida, se ensambla un catálogo sismológico apropiado para así determinar la actividad sísmica de cada una de las fuentes. Para esto se usaron los catálogos del USGS-NEIC (USGS, 2013) y del ISC-GEM (Storchak et al., 2013). Ambos catálogos fueron examinados, realizando las debidas revisiones para determinar la calidad y completitud de la información contenida en cada uno de ellos.

Una vez compilados estos catálogos en un solo, se realizó un análisis de completitud de los eventos para diferentes valores de magnitud umbral. Para esto, se ha seguido el procedimiento propuesto por Tinti y Mulargia (1985) en el cual anualmente se acumulan de manera gráfica el número de eventos que igualan o exceden la magnitud umbral, para determinar el año a partir del cual la pendiente de la curva se mantiene aproximadamente constante. Se dice entonces que el catálogo está completo para la magnitud $\mathrm{M}_{0}$ a partir del año encontrado, y son los eventos dentro de esta ventana de tiempo los que se consideran para el proceso de caracterización y parametrización de las fuentes sismogénicas.

El catálogo utilizado para este estudio consta de eventos registrados dentro de los territorios de Chile y Argentina, pero también incluyendo aquellos ocurridos en el Océano Pacífico y en países fronterizos como Perú y Bolivia, entre $77^{\circ} \mathrm{O}$ y $51.5^{\circ} \mathrm{O}$ de longitud y $58.5^{\circ} \mathrm{S}$ y $14^{\circ} \mathrm{S}$ de latitud. Tras haber seleccionado una magnitud umbral a $\left(M_{0}\right)$ de 4.5 , haber eliminado réplicas y haber realizado en análisis de completitud, el catálogo resultante consta de 8,156 eventos que abarcan el período de tiempo entre el 1 de enero de 1973 y el 31 de diciembre de 2013 (ver Figura 1). En los sismos incluidos en el catálogo se ha encontrado una máxima de $\mathrm{M}_{\mathrm{W}}=8.8$, correspondiente al evento ocurrido en Chile el 27 de febrero de 2010.

La sismicidad de las fuentes se modela considerando un modelo de recurrencia poissoniano, en el cual se asume que los eventos sísmicos ocurren de manera independiente entre sí. En el modelo poissoniano, la actividad de la i-ésima fuente sísmica se especifica en términos de la tasa de excedencia de las magnitudes, $\lambda_{i}(M)$, generadas por esta fuente. La tasa de excedencia de magnitudes mide qué tan frecuentemente se generan temblores con magnitud superior a una específica. La función $\lambda_{\mathrm{i}}(M)$ es una versión modificada de la relación de Gutenberg y Richter, en donde la sismicidad se describe como: 


$$
\lambda(M)=\lambda_{0} \frac{e^{-\beta M}-e^{-\beta M_{u}}}{e^{-\beta M_{0}}-e^{-\beta M_{u}}}
$$

en donde $M_{0}$ es la mínima magnitud o magnitud umbral. $\lambda_{0}, \beta$, y $M_{u}$ son parámetros que definen la tasa de excedencia de cada una de las fuentes sísmicas. Estos parámetros, diferentes para cada fuente, se estiman mediante el procedimiento estadístico de máxima verosimilitud. De esta manera, cada una de las fuentes sismogénicas queda caracterizada por los siguientes de parámetros de sismicidad:

- Recurrencia de magnitudes: se identifica mediante el parámetro $\beta$ que representa la pendiente promedio (en escala logarítmica) de la curva de recurrencia de magnitudes (curva de número de eventos con magnitud mayor que $M$, versus magnitud sísmica $M$ ) en la zona de magnitudes bajas.

- Magnitud máxima $\mathbf{M}_{\mathrm{u}}$ : se estima con base en la máxima longitud de ruptura posible de cada una de las fuentes y en otras características morfotectónicas.

- Tasa de recurrencia de sismos con magnitud mayor que la de umbral $\lambda_{0}$ : corresponde al número promedio de sismos por año con magnitud mayor que $M_{0}$ que ocurren en una determinada fuente.

Una vez determinada la tasa de actividad de cada una de las fuentes sísmicas, es necesario evaluar los efectos que, en términos de intensidad sísmica, produce cada una de ellas en un sitio de interés. Para ello se requiere saber qué intensidad se presentaría en el sitio en cuestión, si en la i-ésima fuente ocurriera un temblor con magnitud dada. Para esto se requiere la definición de uno o varios modelos de atenuación (conocidos también como Ground Motion Prediction Equations, GMPE), que describan de manera apropiada los procesos de transformación que sufren las ondas sísmicas en su propagación por la corteza terrestre, y cómo estos procesos se ven reflejados en la intensidad de movimiento fuerte que ocurre en un sitio específico tras la ocurrencia de un terremoto.

La intensidad de movimiento fuerte se modela como una variable aleatoria con el fin de incorporar de manera racional la incertidumbre asociada a su estimación. En el caso del modelo de amenaza del Cono sur, es de interés la estimación de la aceleración máxima (PGA), así como ordenadas del espectro de respuesta (aceleración espectral). Es usual representar estos parámetros de movimiento fuerte como variables aleatorias distribuidas lognormal, con momentos de probabilidad dados por el modelo de atenuación.

Se seleccionaron los modelos de atenuación propuestos por Sadigh et al. (1997) y Zhao et al. (2006) que dan cuenta de las características del movimiento en roca. Estas relaciones de atenuación fueron asignadas a fuentes intraplaca y de subducción respectivamente.

La Figura 3 muestra las relaciones de atenuación para diferentes mecanismos, magnitudes y ordenadas espectrales de manera ilustrativa (Sadigh et al. 1997 strike-slip: rojo, Sadigh et al. 1997 reverse: amarillo, Zhao et al. 2006 interface: naranja y Zhao et al. 2006 intraslab: verde).

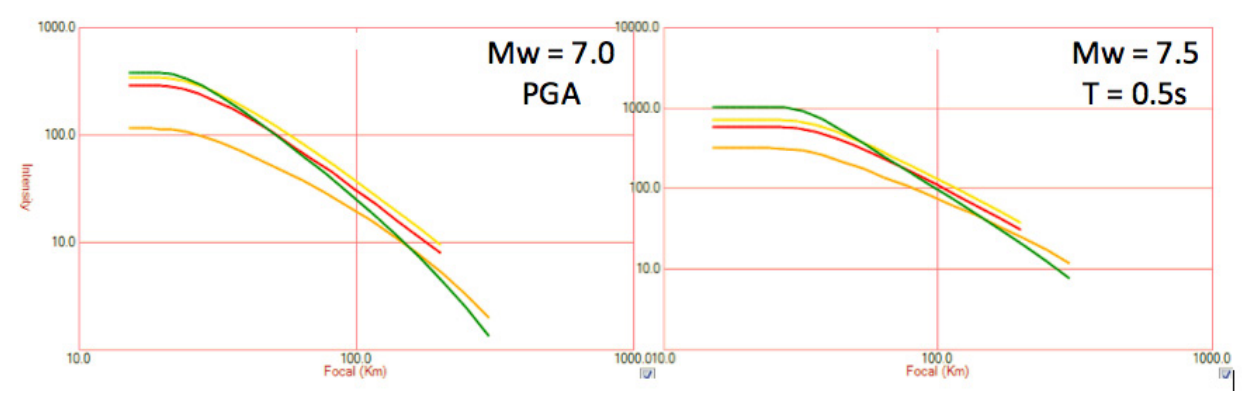

Figura 3. Relaciones de atenuación para diferentes magnitudes y periodos. Adaptado de: Costa et al., 2000; Lavenu et al., 2000; SEGEMAR, 2015; SERNAGEOMIN, 2014. 
Una vez conocidas la sismicidad de cada una de las fuentes y los patrones de atenuación de las ondas generadas en cada una de ellas, puede calcularse la amenaza sísmica considerando la suma de los efectos de la totalidad de las fuentes sísmicas y la distancia entre cada fuente y el sitio donde se encuentran los elementos expuestos de interés. La amenaza, expresada en términos de las tasas de excedencia de intensidades, a, se calcula mediante la siguiente expresión (Ordaz, 2000):

$$
v(a)=\sum_{n=1}^{N} \int_{M_{0}}^{M_{U}}-\frac{\partial \lambda}{\partial M} \operatorname{Pr}\left(A>a \mid M, R_{i}\right) d M
$$

donde la sumatoria abarca la totalidad de las fuentes sismogénicas $N$, y $\operatorname{Pr}\left(A>a \mid M, R_{i}\right)$ es la probabilidad de que la intensidad exceda un cierto valor, dadas la magnitud del sismo, $M$, y la distancia entre la i-ésima fuente y el sitio, $R_{i}$. Las funciones $\lambda_{i}(M)$ son las tasas de actividad de cada una de las fuentes sismogénicas. La integral se realiza desde $M_{0}$ hasta $M_{u}$ lo que indica que se toma en cuenta, para cada fuente sismogénica, la contribución de todas las magnitudes. En el presente estudio $M_{0}$ es igual para todas las fuentes mientras que $M_{u}$ varía en cada caso.

Conviene hacer notar que la ecuación anterior sería exacta si las fuentes sismogénicas fueran puntos. Dado que en realidad son volúmenes los epicentros no sólo pueden ocurrir en los centros de las fuentes sino, con igual probabilidad, en cualquier punto dentro del volumen correspondiente. Se debe tomar en cuenta esta situación subdividiendo las fuentes sismogénicas en este caso en triángulos, en cuyo centro de gravedad se considera concentrada la sismicidad del triángulo. La subdivisión se hace recursivamente hasta alcanzar un tamaño de triángulo suficientemente pequeño como para garantizar la precisión en la integración de la ecuación anterior.

Los cálculos de la amenaza sísmica fueron realizados utilizando el programa de cálculo CRISIS 2014 V1.2 (Ordaz et al., 2014) que permite la obtención de los resultados de amenaza sísmica en manera compatible con la Plataforma CAPRA (Cardona et al., 2012), es decir en términos de un conjunto de escenarios estocásticos para la posterior evaluación probabilista de riesgo sísmico.

\section{Resultados de la amenaza sísmica}

El resultado principal de una evaluación probabilista de amenaza sísmica consiste en la obtención de la curva de excedencia de intensidades. Estas intensidades en el presente estudio corresponden a valores de aceleración espectral en cada sitio de cálculo y entonces, por consiguiente, en cada una de ellas se puede leer la tasa de excedencia (número de veces por año) para diferentes valores. Los resultados se obtienen para cada punto de la malla de análisis por lo que es posible entonces, a partir de estos obtener mapas de amenaza para diferentes períodos de retorno como los que se presentan en esta sección.

Dado que el análisis de amenaza sísmica ha sido realizado para diferentes ordenadas espectrales, seleccionando uno o varios períodos de retorno es posible obtener espectros de amenaza uniforme. Cada uno de estos tiene la particularidad de que, para cada ordenada espectral, se tiene asociado el mismo período de retorno.

La Figura 4 presenta las curvas de excedencia para aceleración máxima del terreno (PGA) en algunas ciudades de Argentina y Chile. La Figura 5 presenta los espectros de amenaza uniforme para diferentes períodos de retorno: $100,250,475,1,000$ y 2,500 años, en estas mismas ciudades.

Así mismo, en la Figura 6 se presentan los mapas de amenaza calculados para aceleración máxima del terreno (PGA) y aceleración espectral para un período estructural de 0.5 segundos, y para períodos de retorno de 500, 1,000 y 2,500 años. 

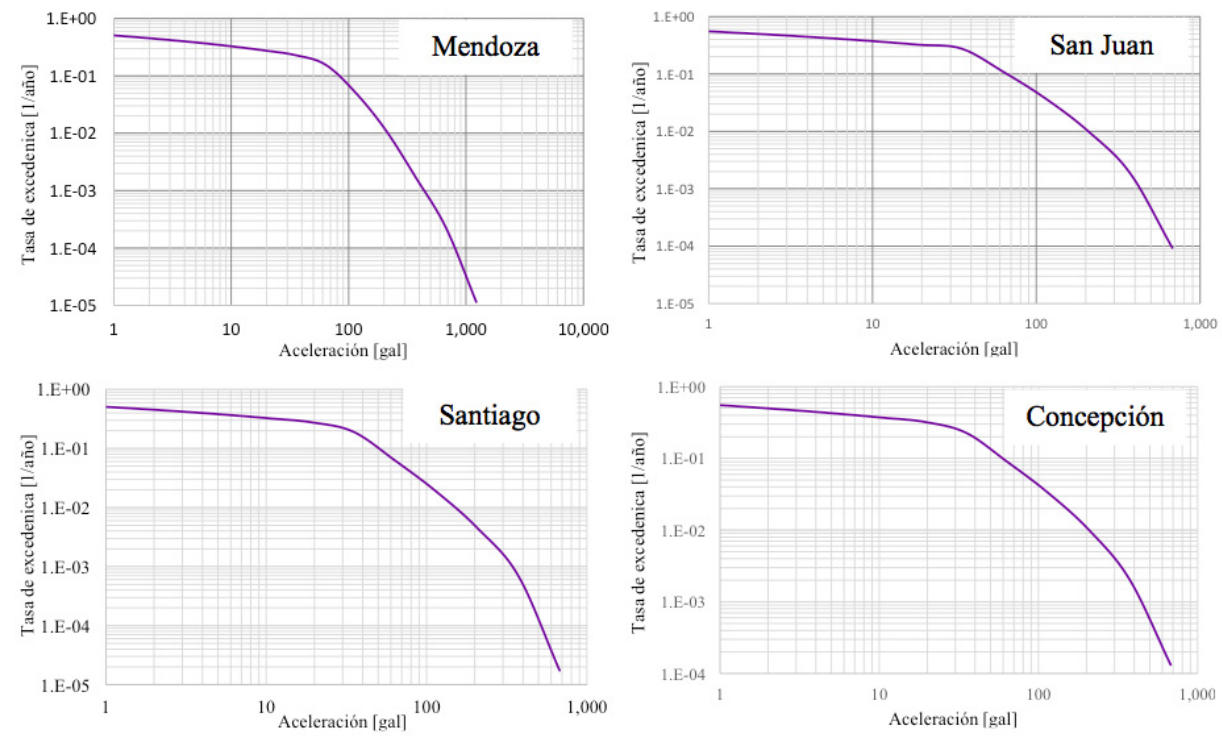

Figura 4. Curvas de amenaza para PGA.
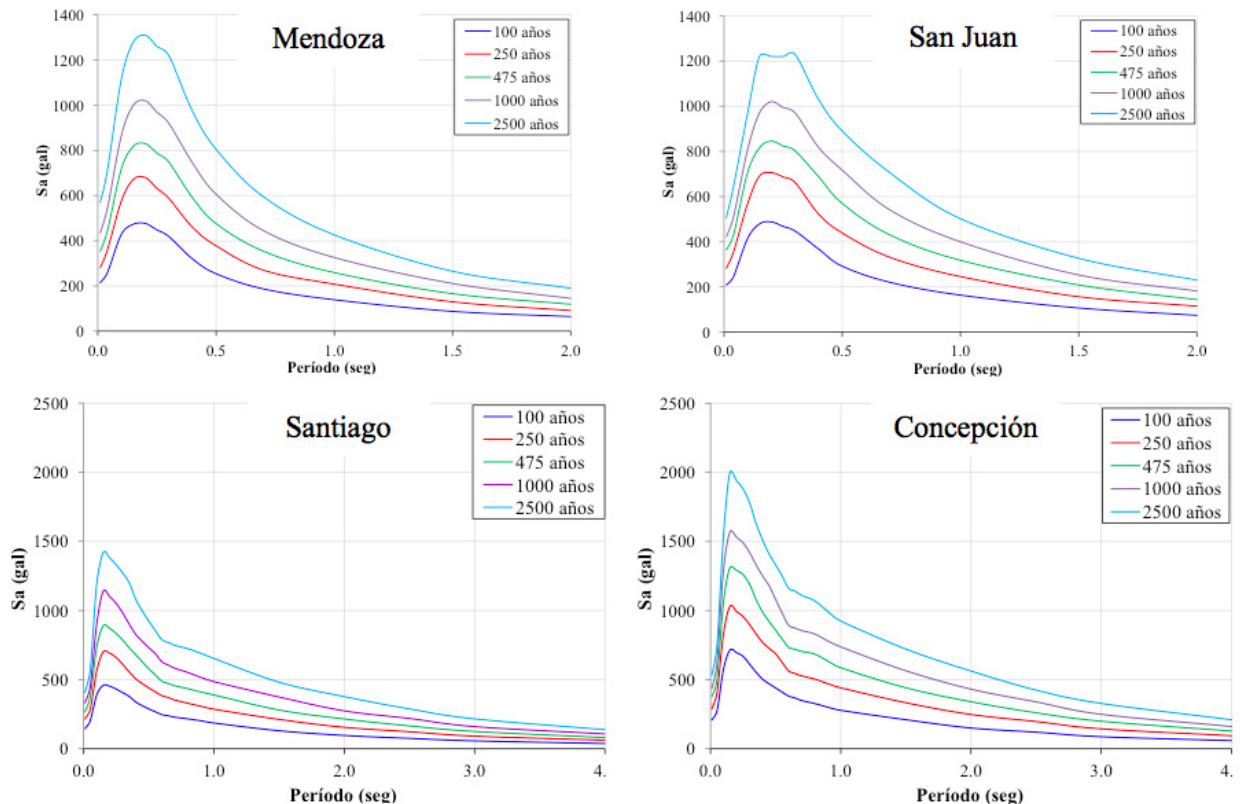

Figura 5. Espectros de amenaza uniforme. 


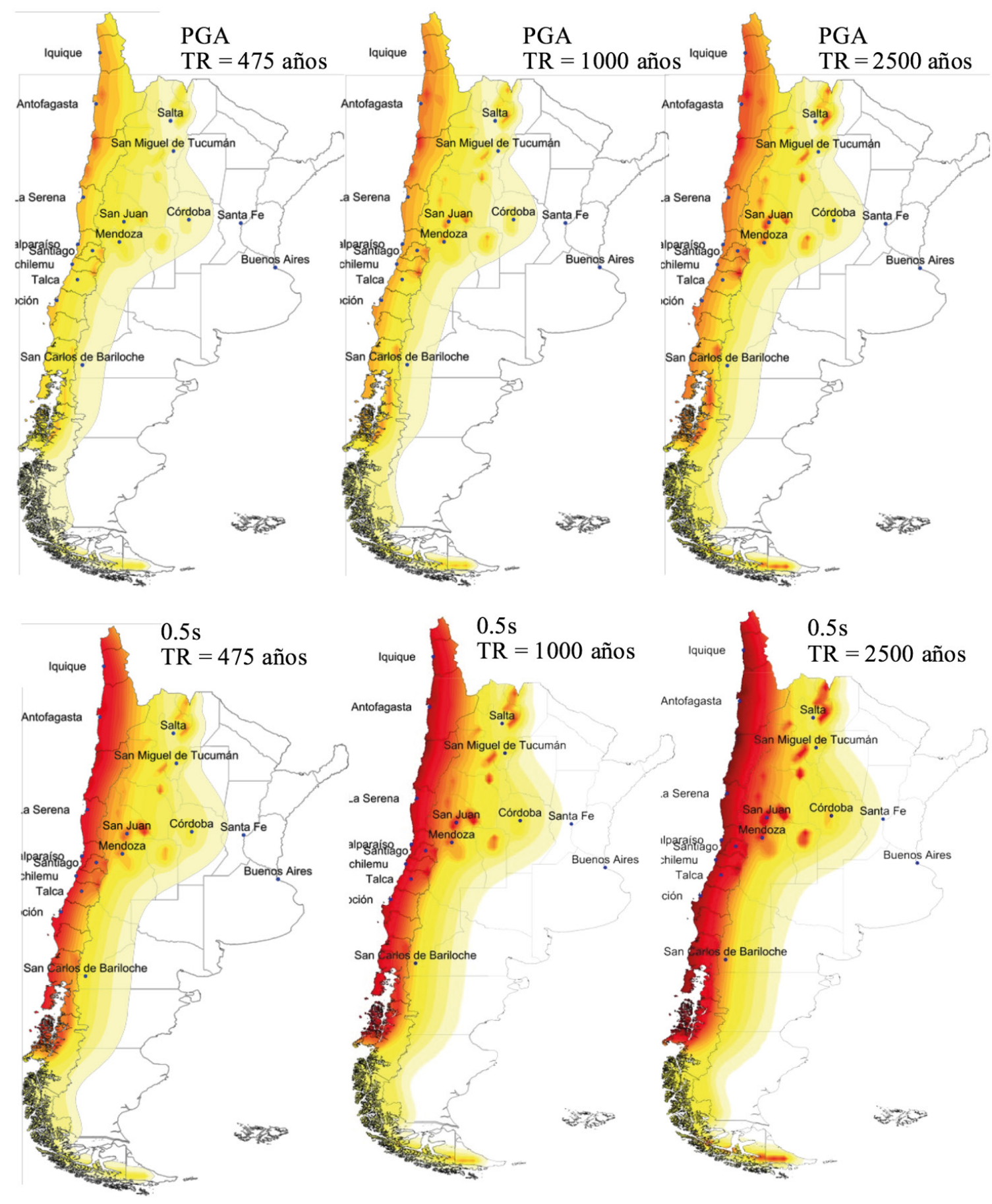

$\begin{array}{lllllllllllll}0 & 150 & 300 & 450 & 600 & 750 & 900 & 1050 & 1200 & 1350 & 1500 & 1650 & 1800\end{array}$

Figura 6. Aceleración para varios periodos estructurales

y periodos de retorno. Escala de aceleración en $\mathrm{cm} / \mathrm{s}^{2}$ 


\section{EVALUACIÓN DEL RIESGO SÍSMICO}

La evaluación probabilista del riesgo sísmico tiene como objetivo estimar la distribución de probabilidad de la pérdida económica que puede presentarse en un conjunto de elementos expuestos, dada la ocurrencia de un terremoto particular. El riesgo se deriva de la combinación de tres componentes principales:

1. La amenaza, la cual se representa por medio de una colección de escenarios, generados de manera estocástica, los cuales representan de manera integral, y en términos de probabilidad, la amenaza sísmica de una región. Cada escenario tiene asociada una frecuencia de ocurrencia y contiene la distribución espacial de parámetros que permiten construir la distribución de probabilidad de las intensidades producidas por su ocurrencia.

2. Los elementos expuestos, que son el conjunto de bienes o activos (construcciones) susceptibles de sufrir daños y producir pérdidas, dada la ocurrencia de los escenarios que definen la amenaza. Estos elementos se caracterizan por su ubicación geográfica, su valor de reposición y la clase estructural a la que pertenecen.

3. La vulnerabilidad, la cual es una característica intrínseca de los elementos expuestos y que caracteriza el comportamiento de la construcción durante la ocurrencia de un sismo. Está definida mediante curvas de vulnerabilidad, las cuales relacionan los momentos de probabilidad (valor esperado y varianza) de la pérdida en el elemento expuesto, como función de la intensidad de movimiento fuerte que ocurra en su ubicación.

El trasfondo teórico de la representación probabilista de la amenaza sísmica para la evaluación del riesgo se presenta en Bernal (2014), Bernal y Cardona (2015), y Ordaz (2000)

\section{Modelo de activos expuestos}

En general, puede definirse como elemento expuesto cualquier objeto, geográficamente referenciado, que sea susceptible de sufrir algún tipo de afectación por causa de la ocurrencia de un evento de amenaza. Una vez identificado cada uno de los elementos expuestos, es necesario asignar como mínimo un valor económico de reposición, una ocupación humana y una definición de su vulnerabilidad.

Los elementos expuestos son fundamentales dentro del análisis de riesgo, debido a que comprenden los objetos sobre los cuales se evalúan las pérdidas, es decir, son la fuente de las pérdidas potenciales debido al hecho de estar expuestos a una amenaza y ser susceptibles de sufrir un daño. En términos matemáticos, los elementos expuestos proveen individualmente el valor absoluto máximo posible de la pérdida en su ubicación, así como los sumandos de la función de densidad de probabilidad de la pérdida de un escenario de amenaza. Es decir, son en primera medida el elemento integrador de la amenaza y la vulnerabilidad en su ubicación, y en segunda medida, el elemento integrador de las pérdidas totales para un escenario. Su adecuada caracterización es de gran importancia para la adecuada estimación de las pérdidas.

Adicionalmente, los elementos expuestos tienen implícito un componente asociado a la responsabilidad sobre las pérdidas. Si, por ejemplo, los elementos expuestos de un análisis de riesgo corresponden a centros públicos de salud, entonces las pérdidas causadas por la ocurrencia de fenómenos de amenaza serán responsabilidad de la institución de salud pública correspondiente. En caso que los elementos expuestos sean, por ejemplo, las viviendas de los estratos socio-económicos más bajos, estamos hablando de una responsabilidad fiscal del estado, dada la imposibilidad de los propietarios de dichas viviendas privadas de responder a la situación de desastre. Es por esto importante determinar la responsabilidad sobre las pérdidas directamente en la definición de los elementos expuestos. Por esta razón los elementos expuestos se agrupan en portafolios, los cuales permiten determinar de manera general dicha responsabilidad. Es posible entonces contar dentro de una misma evaluación del riesgo con portafolios de, por ejemplo, centros públicos de salud, centros educativos, viviendas, edificaciones institucionales, infraestructura vial, infraestructura de comunicaciones, etc. En definitiva, deben definirse tantos portafolios como haya entidades o instituciones responsables sobre las pérdidas.

La información de exposición frente a fenómenos naturales corresponde al inventario de bienes inmuebles e infraestructura que pueden ser afectados y se expresa en términos de activos y de población. La Figura 7 presenta el procedimiento general para desarrollar un modelo simplificado de activos expuestos para el país. 


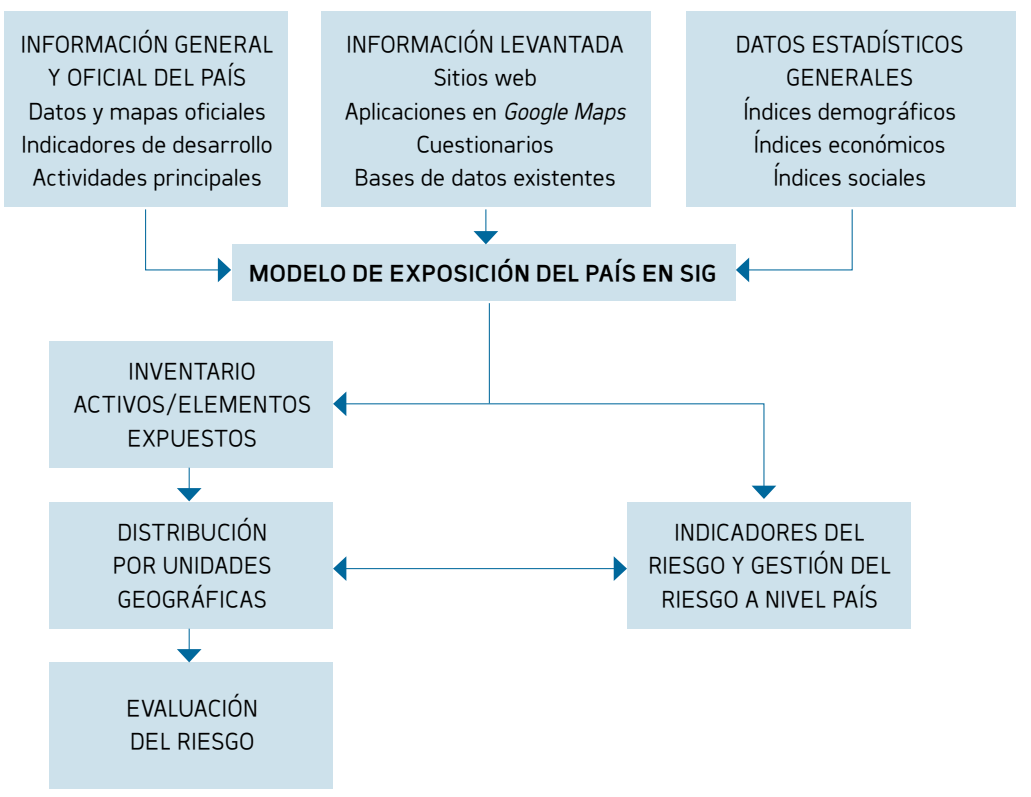

Figura 7. Modelo de activos expuestos

Los indicadores de exposición se desarrollan con el fin de representar la exposición física, económica y humana de un país o una ciudad en términos geográficos. Para esto se clasifican en las siguientes categorías principales:

- Construcciones urbanas y rurales.

- Infraestructura urbana relevante para las principales ciudades del país.

- Infraestructura relevante a nivel nacional.

Con el objeto de identificar el valor expuesto de construcciones convencionales (edificaciones) en estos países, se realizó un inventario lo más exhaustivo posible de los centros urbanos correspondientes a cada una de las entidades subnacionales. Los tipos de edificación se estiman según los sectores económicos presentes y las necesidades básicas de la población como son el servicio de salud y educación, entre otros. La composición (uso) y tamaño $\left(\mathrm{m}^{2}\right)$ de las construcciones se estima utilizando el censo de vivienda desagregado según los siguientes grupos de uso:

(a) Residencial PB: capacidad económica baja (Res PB)

(b) Residencial PM: capacidad económica media (Res PM)

(c) Residencial PA: capacidad económica alta (Res PA)

(d) Comercial (Com)

(e) Industrial (Ind)

(f) Salud privada (SalPri)

(g) Educación privada (EduPri)

(h) Salud pública (SalPub)

(i) Educación pública (EduPub)

(j) Gubernamentales (Gob)

Para la elaboración de este análisis es necesario estimar el área construida por habitante, los tipos de usos y los niveles de complejidad, el valor económico de cada metro cuadrado de desarrollo por tipo de uso y nivel de complejidad, y el nivel de ocupación por cada tipo de desarrollo en un escenario dado, expresado en términos de metros cuadrados de área construida por tipo de uso y nivel de complejidad.

Con el objeto de identificar el valor expuesto de infraestructura urbana en toda la extensión de ambos países, y utilizando el inventario de los centros urbanos correspondientes a cada una de las entidades subnacionales, se realizó una estimación de la cobertura de servicios públicos y valoración de las redes (acueducto, alcantarillado, comunicación), puentes, aeropuertos y puertos. 
Las coberturas de servicios públicos e infraestructura de transporte, se estima a partir de información incluida en el censo de vivienda bajo las siguientes categorías:
(a) Puentes urbanos
(b) Aeropuertos
(c) Puertos
(d) Subestaciones de energía más redes anexas
(e) Subestaciones de comunicaciones más antenas
(f) Redes de acueducto y alcantarillado
(g) Tanques y plantas de acueducto y alcantarillado
(h) Redes de gas

Con el objeto de cuantificar el valor expuesto de infraestructura nacional se utilizó el inventario de entidades subnacionales junto con los centros poblados y tipos de servicios que disponen. Con base en esto se realizó una estimación de la cobertura de servicios y valoración de los componentes de infraestructura tales como hidroeléctricas, redes de interconexión nacional, líneas de transporte de hidrocarburos y redes viales nacionales. La infraestructura nacional se clasifica en las siguientes categorías:
(a) Vías red primaria
(b) Vías red secundaria
(c) Hidroeléctricas
(d) Presas
(e) Plantas térmicas
(f) Subestaciones de energía más redes anexas
(g) Subestaciones de comunicaciones más antenas
(h) Subestaciones de combustible y gas más redes anexas

La asignación de valores sobre la infraestructura descrita anteriormente se realiza bajo el estimativo de cobertura de la población con los servicios relacionados a cada tipo de infraestructura, la producción energética del país, el número de líneas móviles y fijas y del nivel de hidrocarburos explotados. Los anteriores valores se ubican geográficamente respecto de la densidad de población y los centros de producción. A continuación, se presentan mapas con la distribución del valor expuesto total por entidad subnacional, para edificaciones, infraestructura urbana e infraestructura nacional para ambos países.
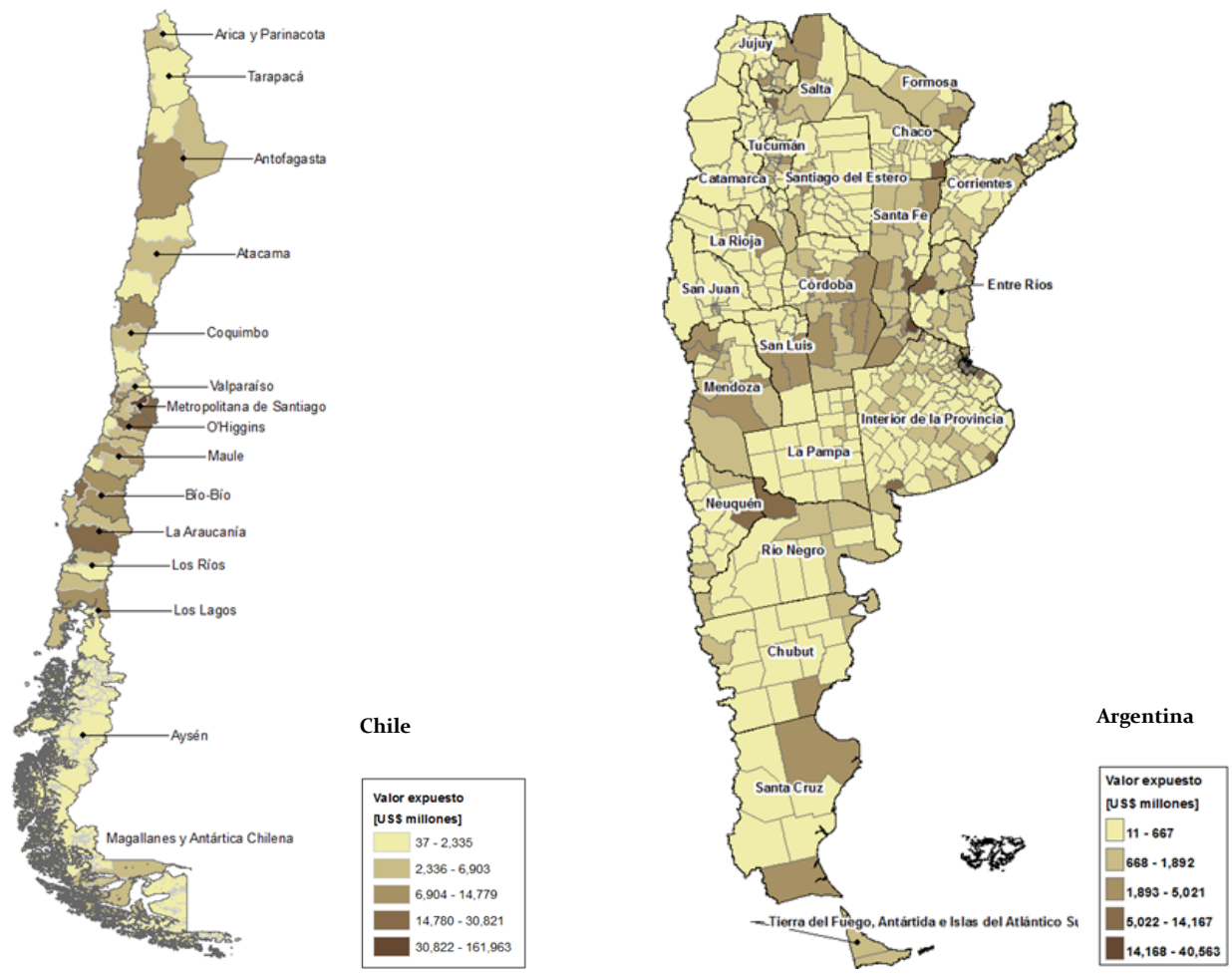


\section{Vulnerabilidad física ante la amenaza sísmica}

La vulnerabilidad de los elementos expuestos se caracteriza mediante funciones de vulnerabilidad, las cuales definen el comportamiento de una construcción durante la ocurrencia de un terremoto. Las funciones de vulnerabilidad indican la distribución de probabilidad de la pérdida (p) como función de la intensidad producida durante un escenario específico, es decir, condicional a la ocurrencia de un nivel de intensidad (a) en la ubicación del elemento expuesto. La pérdida $p$ generalmente se entiende como la pérdida relativa al valor de reposición del elemento expuesto. La pérdida se asume típicamente como distribuida Beta.

La vulnerabilidad sísmica relaciona entonces cualquier medida de intensidad de movimiento fuerte con la pérdida relativa en el elemento físico expuesto a dicha intensidad. Por ejemplo, para el caso de construcciones típicas de edificios de varios pisos, la intensidad sísmica que mejor se correlaciona con los daños esperados es la deriva o distorsión angular de entrepisos (relacionada con la deformación de la estructura ante las fuerzas sísmicas). Para otro tipo de construcciones, como edificaciones menores en mampostería o adobe, se utiliza principalmente la aceleración máxima del terreno como parámetro de correlación con el daño. En otros casos como los sistemas de tuberías enterradas, resulta más conveniente utilizar como parámetro de intensidad como la velocidad máxima del terreno

Primero se realiza una tipificación de los tipos constructivos más representativos, con base en la información existente y criterios aportados a nivel local. Luego se hace el cálculo de las funciones de vulnerabilidad de estos tipos constructivos; para esto se utilizaron funciones aplicables ya publicadas según experiencias previas. Finalmente, se hace una asignación de un tipo constructivo característico a cada uno de los elementos que conforman el inventario de activos expuestos y asignación de la función de vulnerabilidad correspondiente.

La Figura 9 muestra las funciones de vulnerabilidad utilizadas para las edificaciones de ambos países, mientras que la Figura 10 muestra las funciones utilizadas para la infraestructura de ambos países.

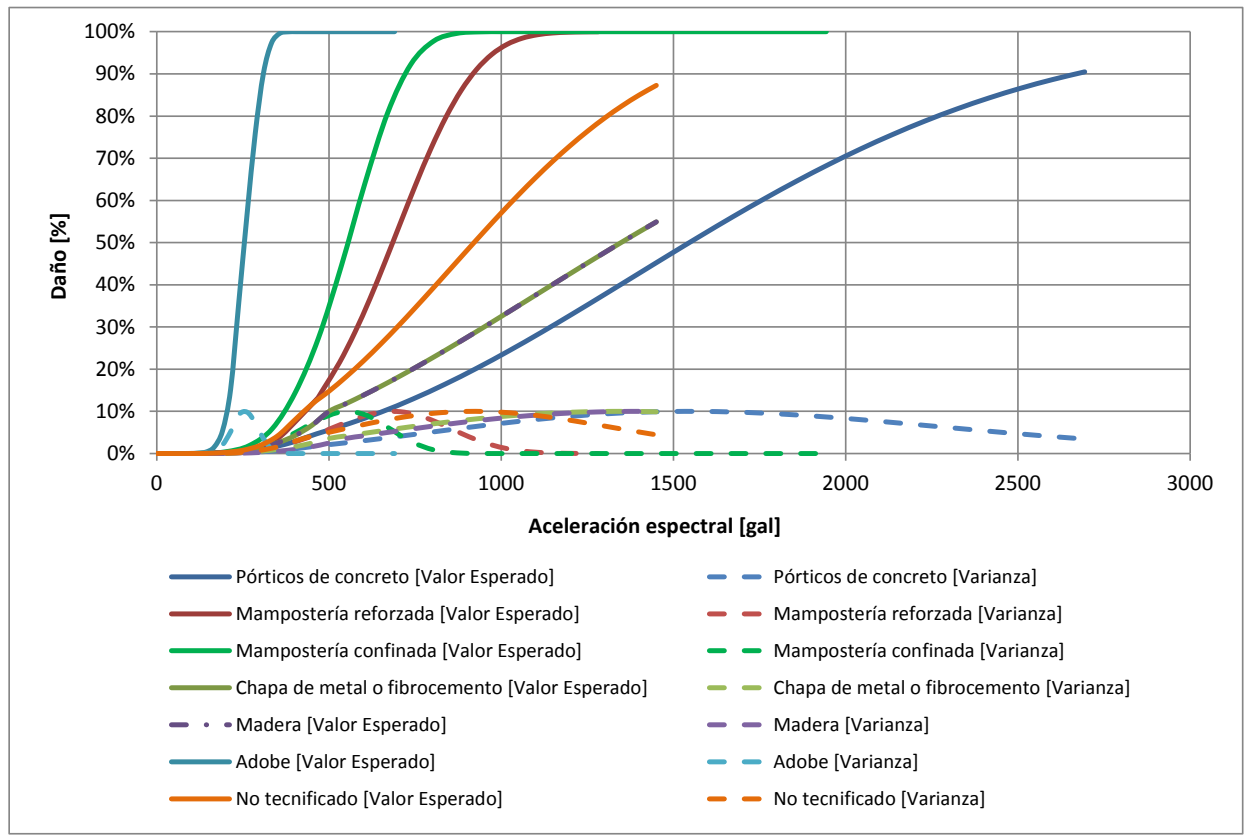

Figura 9. Funciones de vulnerabilidad para construcciones convencionales. 


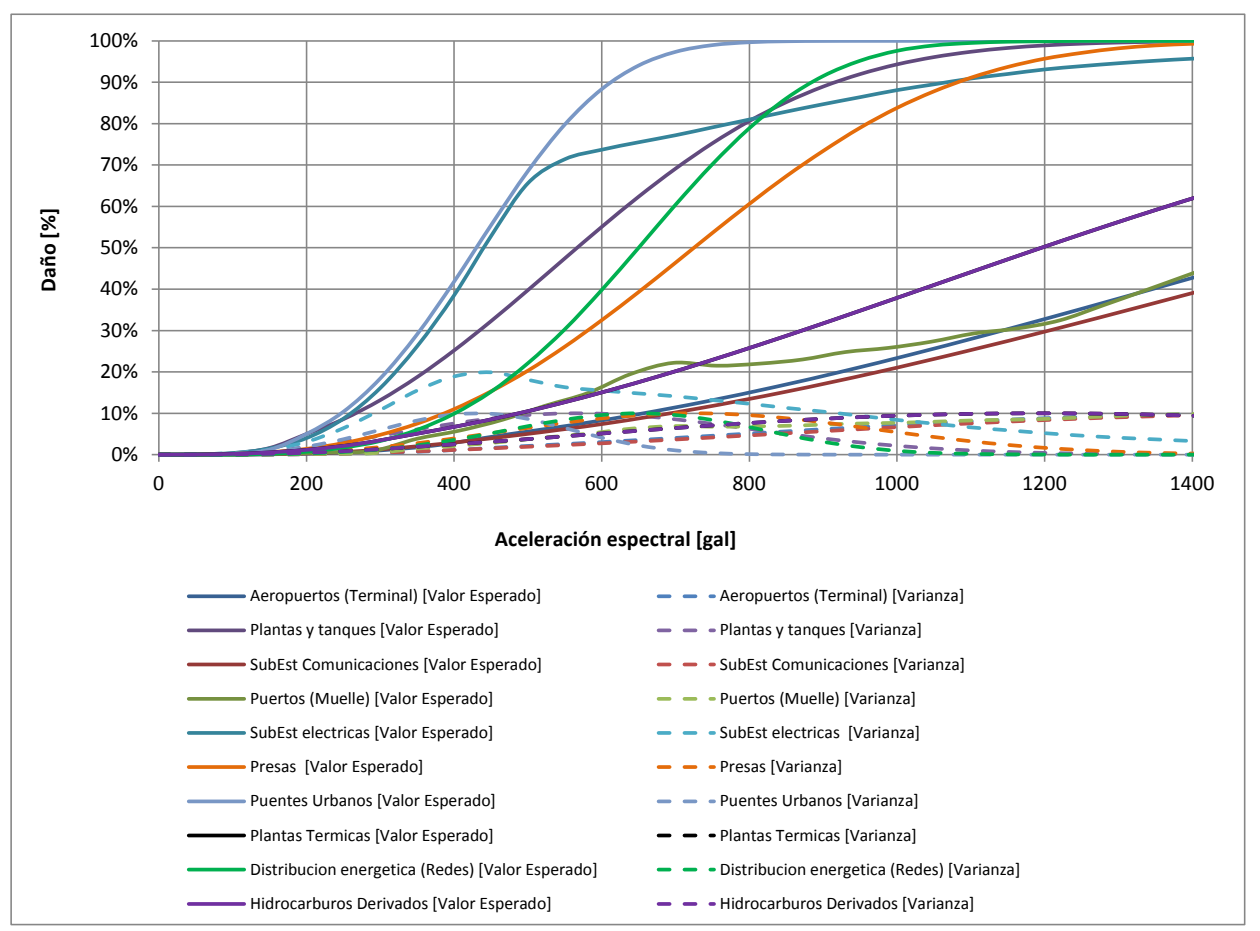

Figura 10. Funciones de vulnerabilidad para infraestructura.

\section{Evaluación probabilista del riesgo sísmico}

El riesgo se determina mediante la convolución entre la amenaza y la vulnerabilidad, en la ubicación ocupada por los elementos expuestos y para la totalidad de los mismos. Como se ha mencionado anteriormente, en el cálculo probabilista de las pérdidas la amenaza y la vulnerabilidad están representadas también de manera probabilista. La amenaza tiene una representación probabilista tanto temporal como espacial. La ocurrencia en el tiempo de los escenarios de amenaza se modela como un proceso de Poisson, lo que conlleva a modelar mediante una distribución de probabilidad exponencial el tiempo que transcurre entre los escenarios. Por otra parte, la medida de intensidad de movimiento fuerte en cada ubicación modela como una variable aleatoria con distribución Lognormal, definida por sus dos primeros momentos de probabilidad (su mediana y la desviación estándar del logaritmo natural). La vulnerabilidad se define también por medio de dos momentos de probabilidad los cuales define la distribución de probabilidad Beta asignada a la pérdida.

Las incertidumbres asociadas a la amenaza y a la vulnerabilidad son entonces definidas en su propia naturaleza; temporal y espacial para la amenaza y dependiente de la intensidad para la vulnerabilidad. De esta manera, el principal resultado del modelo es una distribución de probabilidad específica de la pérdida para cada escenario. De acuerdo con el procedimiento analítico propuesto por Ordaz (2000), la función de densidad de probabilidad de la pérdida en el elemento $j$, condicional a la ocurrencia del escenario $i, f\left(p_{j} \mid E_{i}\right)$, se calcula como:

$$
f\left(p_{j} \mid E_{i}\right)=\int_{0}^{\infty} f\left(p_{j} \mid s\right) f\left(s \mid E_{i}\right) d s
$$

en donde $f\left(p_{j} \mid E_{i}\right)$ es la función de densidad de probabilidad de la pérdida en el elemento $j_{\text {, }}$ condicional a la ocurrencia del escenario $i$, el primer término de la integral tiene que ver con la vulnerabilidad $\left(f\left(p_{j} \mid s\right)\right.$, la densidad de probabilidad de la pérdida dada una intensidad $s$ ) y la segunda parte tiene que ver con la amenaza $\left(f\left(s \mid E_{i}\right)\right.$, la densidad de probabilidad de la intensidad, dada la ocurrencia de un escenario). 
La pérdida total del escenario $i$ corresponde a la suma de todas las pérdidas individuales $\left(p_{j}\right)$ de los elementos expuestos. Dado que los sumandos son variables aleatorias, debe usarse la aritmética correcta para determinar la pérdida del escenario $i$. La suma se hace sobre los momentos de probabilidad de las variables $p_{j}$ como se indica a continuación.

$$
\begin{aligned}
E\left(p \mid E_{i}\right) & =\sum_{j=1}^{N E} E\left(p_{j}\right) \\
\sigma^{2}\left(p \mid E_{i}\right) & =\sum_{j=1}^{N E} \sigma^{2}\left(p_{j}\right)+2 \sum_{\substack{k=1 \\
k<j}}^{N E-1} \sum_{j=2}^{N E} \operatorname{cov}\left(p_{k}, p_{j}\right)
\end{aligned}
$$

en donde $E\left(p \mid E_{i}\right)$ es el valor esperado de la pérdida del escenario $i$, 2(p|Ei) es su varianza, $N E$ es el número total de elementos expuestos, $E\left(p_{j}\right)$ es el valor esperado de la pérdida en el elemento $j, \sigma^{2}\left(p_{j}\right)$ es su varianza, y $\operatorname{cov}\left(p_{k}, p_{j}\right)$ es la covarianza de la pérdida de dos elementos expuestos diferentes.

El riesgo es comúnmente descrito mediante la llamada curva de excedencia de pérdidas la cual especifica las frecuencias de ocurrencia de eventos en que se exceda un valor específico de pérdidas. Esta frecuencia anual de excedencia se conoce también como tasa de excedencia, y se calcula de la siguiente manera:

$$
v(p)=\sum_{i=1}^{N} \operatorname{Pr}\left(P>p E_{\mathrm{i}}\right) \cdot F_{A}\left(E_{\mathrm{i}}\right)
$$

en donde $v(p)$ es la tasa de excedencia de la pérdida $p, N$ es el número total de escenarios de amenaza, $F_{A}\left(E_{i}\right)$ es la frecuencia anual de ocurrencia del escenario $i$, y $\operatorname{Pr}\left(P>p \mid E_{i}\right)$ es la probabilidad de que $p$ sea excedida dado que ocurrió el escenario $i$. La suma cubre todos los escenarios de amenaza que pueden generar pérdidas. El inverso de $v(p)$ es el periodo de retorno de la pérdida $p$, identificado como Tr. La curva de excedencia de pérdidas contiene toda la información necesaria para caracterizar, en términos de probabilidad, el proceso de ocurrencia de las pérdidas asociadas a los eventos.

Es importante hacer notar que la curva de excedencia de pérdidas no tiene incertidumbre. Es común asociar los resultados de un modelo probabilista de riesgo a un nivel de confianza o de variabilidad de los mismos. Pues bien, las tasas de excedencia calculadas en un modelo probabilista de riesgo consideran directamente la incertidumbre en las pérdidas, por lo cual dichas tasas de excedencia no pueden tener una incertidumbre o variabilidad propia. Esto debido a que, en términos matemáticos, son una medida de probabilidad calculada para una cantidad de pérdida específica. En el contexto de la modelación del riesgo, la pérdida es la variable aleatoria, no las tasas anuales de excedencia.

A partir de la curva de excedencia de pérdidas es posible obtener diversas métricas del riesgo, las cuales son útiles para diferentes fines dentro de la toma de decisiones y la gestión del riesgo. La Pérdida Anual Esperada (PAE) es el valor esperado de la pérdida anual, también llamada prima pura en el sector de los seguros, ya que es la anualización de todas las pérdidas que se tendrán a futuro, y por lo tanto equivaldría al valor que se tendría que pagar cada año para cubrir dichas pérdidas. En el contexto del riesgo de desastres es práctica común expresar la PAE al millar y no en porcentaje ya que estos valores suelen ser pequeños y al verlo en porcentaje este puede ser desestimado por el lector. Se considera la métrica más robusta y a la vez insensible a las incertidumbres inherentes en el análisis debido a que determina la esperanza matemática de las pérdidas en una base anual, y no su incertidumbre (Marulanda, 2013). Se calcula como:

$$
P A E=\int_{0}^{\infty} v(p) d p \quad \text { ó } \quad P A E=\sum_{i=1}^{N} E\left(P E_{\mathrm{i}}\right) F_{A}\left(E_{\mathrm{i}}\right)
$$


Por otro lado, la Pérdida Máxima Probable (PML) corresponde a una pérdida que ocurre poco frecuentemente, es decir, que está asociada a un periodo de retorno grande. En realidad, la PML es una curva que relaciona las pérdidas a su correspondiente periodo de retorno. No obstante, es práctica común definir la PML como un único valor. La selección del periodo de retorno de la PML depende exclusivamente de la aversión al riego del tomador de decisiones. Por ejemplo, en la industria aseguradora el periodo de retorno de la PML se toma usualmente entre 200 y 1500 años.

Finalmente, asumiendo que el proceso de ocurrencia de las pérdidas en el tiempo sigue un proceso de Poisson, es posible determinar la probabilidad de alcanzar o exceder un nivel de pérdida dado, en un periodo de exposición particular:

$$
\operatorname{Pr}(P>p)_{T}=1-e^{-v(p) T}
$$

en donde $\operatorname{Pr}(P>p)_{T}$ es la probabilidad de excedencia de la pérdida $p$, en el lapso de tiempo $T$ (dado en años).

\section{Resultados}

La Tabla 1 presenta la información consolidada a nivel de ambos países, incluyendo el valor expuesto total, la PAE absoluta (en valor monetario) y al millar (relativa al valor expuesto) y la PML para diferentes períodos de retorno.

\begin{tabular}{|c|c|c|c|c|}
\hline \multirow[b]{2}{*}{ Valor expuesto } & \multicolumn{2}{|c|}{ Chile } & \multicolumn{2}{|c|}{ Argentina } \\
\hline & US $\$ \times 10^{6}$ & 481,840 & US $\$ \times 10^{6}$ & 697,464 \\
\hline \multirow{2}{*}{ Pérdida anual esperada } & US $\$ \times 10^{6}$ & 2,231 & US $\$ \times 10^{6}$ & 199 \\
\hline & $\%$ & 4.6 & $\%$ & 0.3 \\
\hline \multicolumn{5}{|c|}{ PML } \\
\hline Periodo retorno & \multicolumn{2}{|c|}{ Pérdida } & \multicolumn{2}{|c|}{ Pérdida } \\
\hline Años & US $\$ \times 10^{6}$ & $\%$ & US $\$ \times 10^{6}$ & $\%$ \\
\hline 50 & 14,815 & 3.1 & 1,332 & 0.2 \\
\hline 100 & 23,354 & 4.8 & 2,004 & 0.3 \\
\hline 250 & 37,274 & 7.7 & 3,071 & 0.4 \\
\hline 500 & 48,439 & 10.1 & 4,108 & 0.6 \\
\hline 1000 & 59,806 & 12.4 & 5,381 & 0.8 \\
\hline
\end{tabular}

Tabla 1. Pérdidas globales en Chile y Argentina.

En la tabla anterior se puede observar que Argentina tiene un valor expuesto mayor al de Chile ( $43 \%$ mayor), pero por el contrario la PAE es mucho menor ( $91 \%$ menor), lo que hace que la diferencia en este valor expresado por mil (\%o) se acentúe aún más (es $93 \%$ menor). Los valores de PML también son considerablemente menores que los de Chile (también alrededor de un 93\% menor). Estas diferencias se explican, primero, porque Chile es un país con mucha menor extensión que Argentina, y segundo, porque se encuentra mucho más cercano a la subducción de las placas de Nazca y Suramérica, lo que en conjunto hace que la totalidad del país esté bajo amenaza sísmica alta. Argentina, por otro lado, al tener una gran extensión y al estar más alejado de la subducción, tiene casi la mitad de su territorio en amenaza baja o nula.

Adicionalmente, la Figura 11 muestra los resultados en términos de la curva de PML, la curva de excedencia de pérdidas y las curvas de probabilidad de quiebra, para cada país. Estas curvas ilustran lo mencionado anteriormente, evidenciando mayores pérdidas para Chile. 

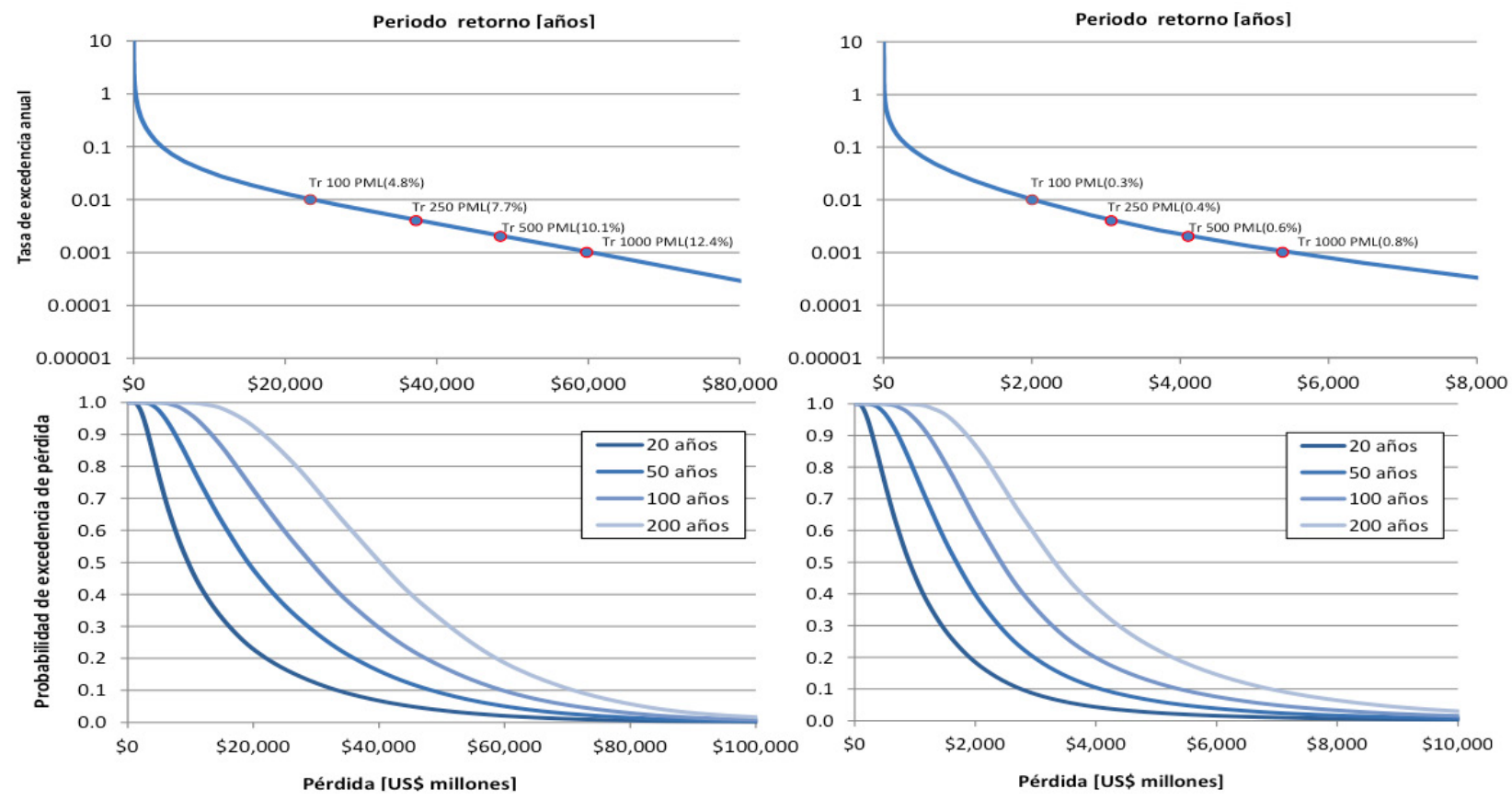

Figura 11. Curvas de excedencia de pérdidas (arriba) y de probabilidad de quiebra (abajo), para Chile (izquierda) y Argentina (derecha)

A continuación, se muestran los resultados de las pérdidas anuales esperadas desagregadas por entidad subnacional para ambos países; la Figura 12 muestra la PAE en valores monetarios absolutos, mientras que la Figura 13 muestra la PAE relativa al valor expuesto.
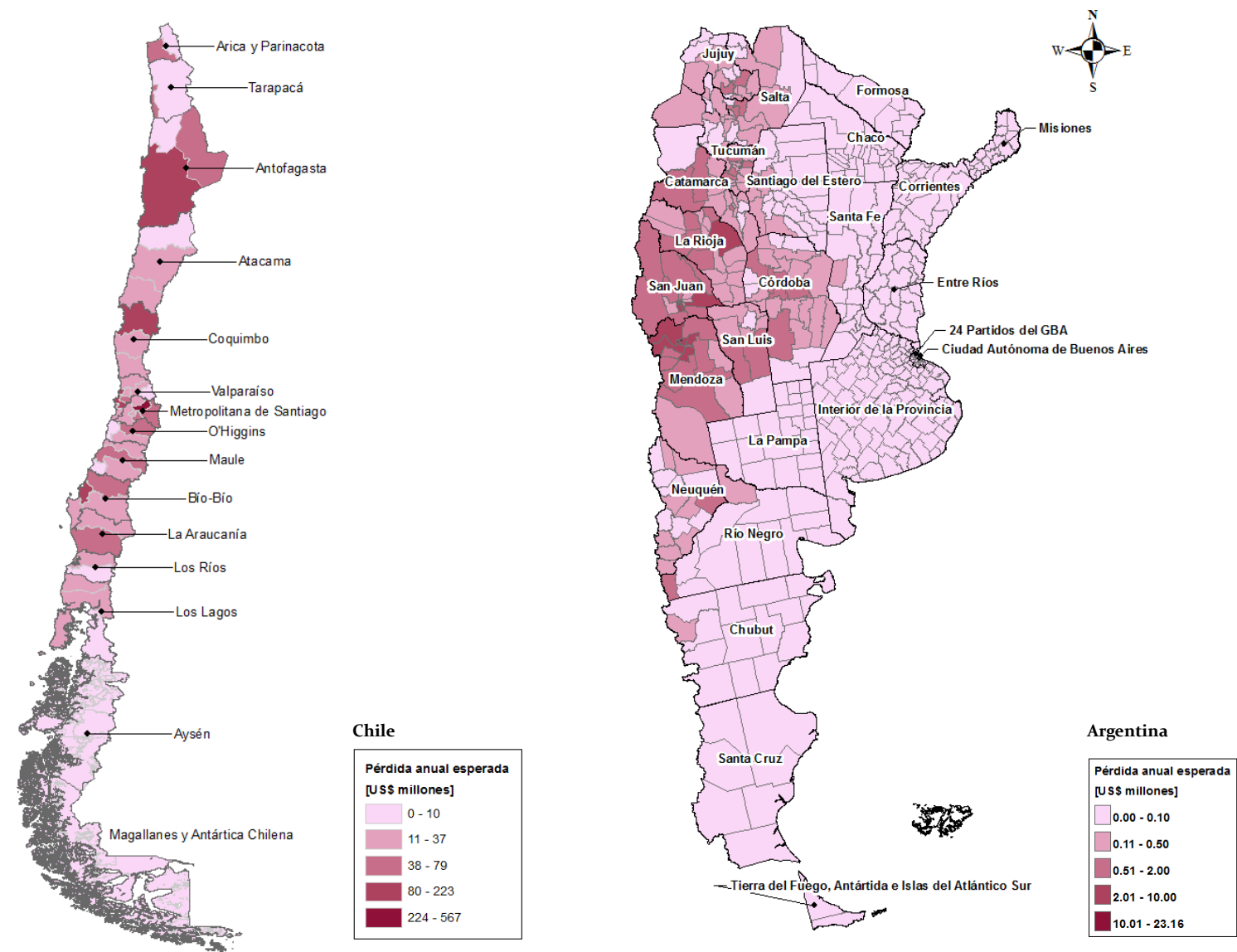

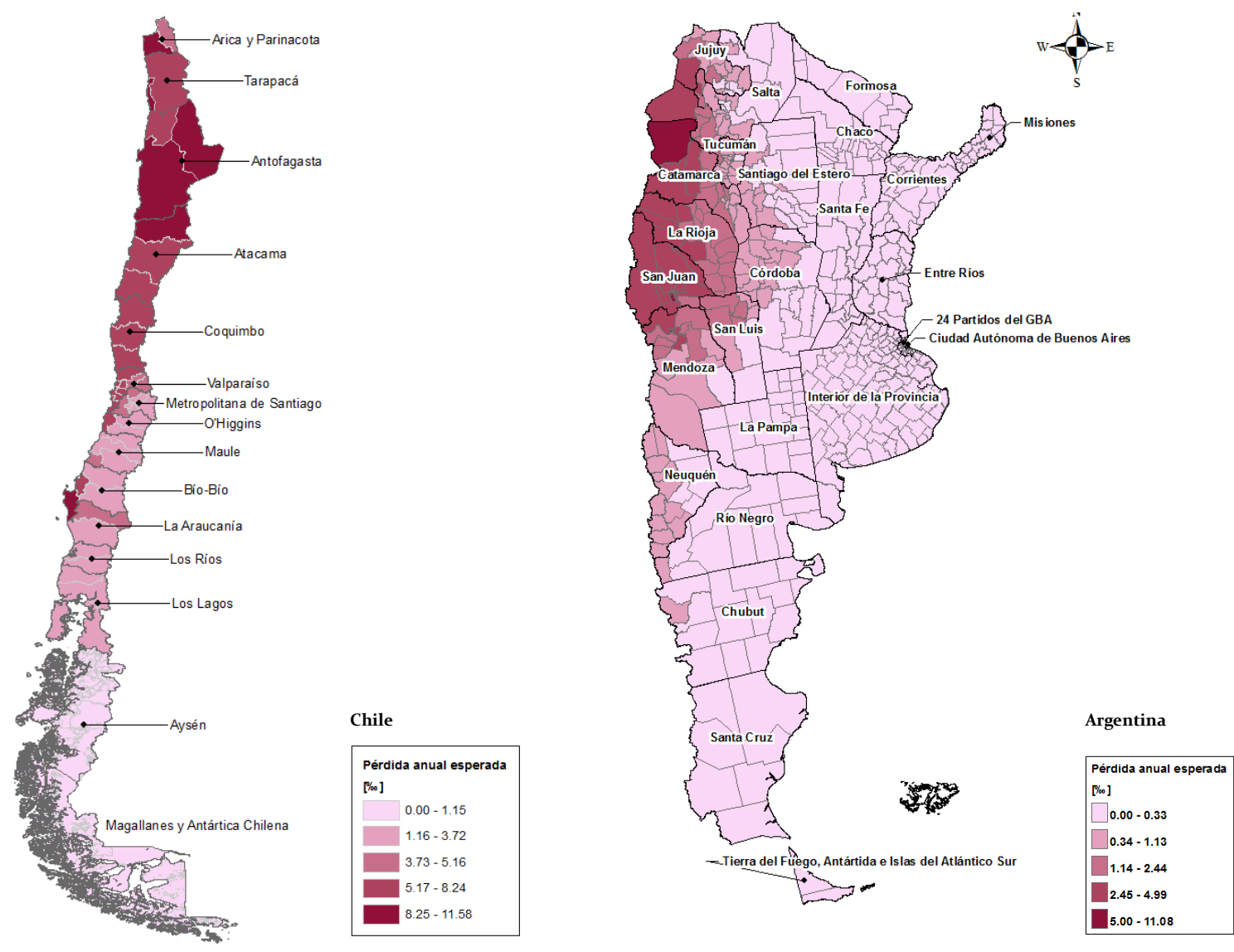

Figura 13. Pérdida anual esperada relativa al valor expuestos para Chile y Argentina

\section{CASO DE ESTUDIO DE RIESGO SÍSMICO EN MENDOZA}

La evaluación probabilista del riesgo presentada anteriormente fue aplicada a una escala nacional; la misma metodología puede ser aplicada a un nivel regional, por ejemplo, para una municipalidad. En este capítulo se presentan el modelo y los resultados de riesgo sísmico aplicados en una escala de mayor resolución para la ciudad de Mendoza, Argentina.

\section{Caracterización de elementos expuestos}

Con el apoyo de la Dirección de Catastro de Mendoza, la Agencia Provincial de Ordenamiento Territorial-APOT, y el Sistema de Información Ambiental Territorial-SIAT, fue posible obtener información general de la ciudad de Mendoza asociada a parámetros como la altura de las edificaciones, zonificación de usos, nivel socio-económico y nivel de sismo-resistencia, con lo cual se conformó una base de datos de elementos expuestos, edificio por edificio, con las características necesarias para la evaluación del riesgo. La Figura 14 muestra la distribución de los sistemas estructurales y el valor expuesto de reposición para las edificaciones mendocinas.

\section{Vulnerabilidad de las edificaciones expuestas}

Tras la caracterización de elementos expuestos, se agruparon los mismos en clases estructurales que dependen del sistema estructural, la altura y si es o no sismorresistente. Los sistemas estructurales existentes incluyen adobe, mampostería confinada, mampostería simple, pórticos de concreto resistente a momento, desecho y madera. Por otro lado, se han definido tres grupos de altura de las edificaciones, como sigue: baja hasta 2 pisos, media hasta 5 pisos y alta de 6 pisos 0 más. Finalmente se utilizó información de la microzonificación sísmica de Mendoza (INPRES, 1989) en cuanto a la distribución porcentual de construcciones sismorresistentes y no sismorresistentes por distrito. 


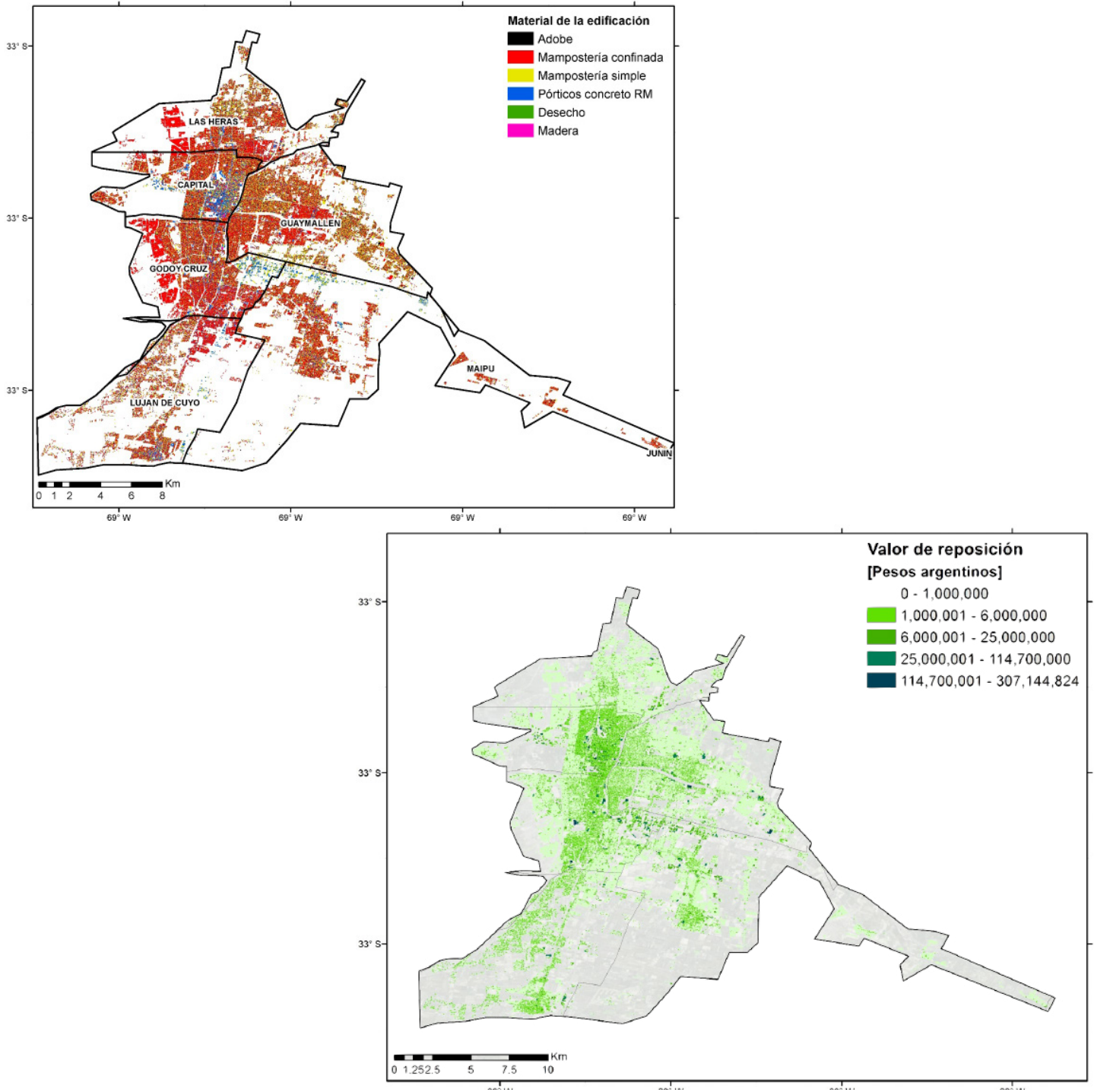

Figura 14. Sistema estructural (arriba) y valor de reposición (abajo) de las edificaciones de Mendoza

La Figura 15 muestra todas las funciones de vulnerabilidad; de allí se puede notar que las construcciones en adobe en general son las más vulnerables, le siguen las de mampostería simple, mampostería confinada, madera y finalmente los pórticos de concreto, resistentes a momento.

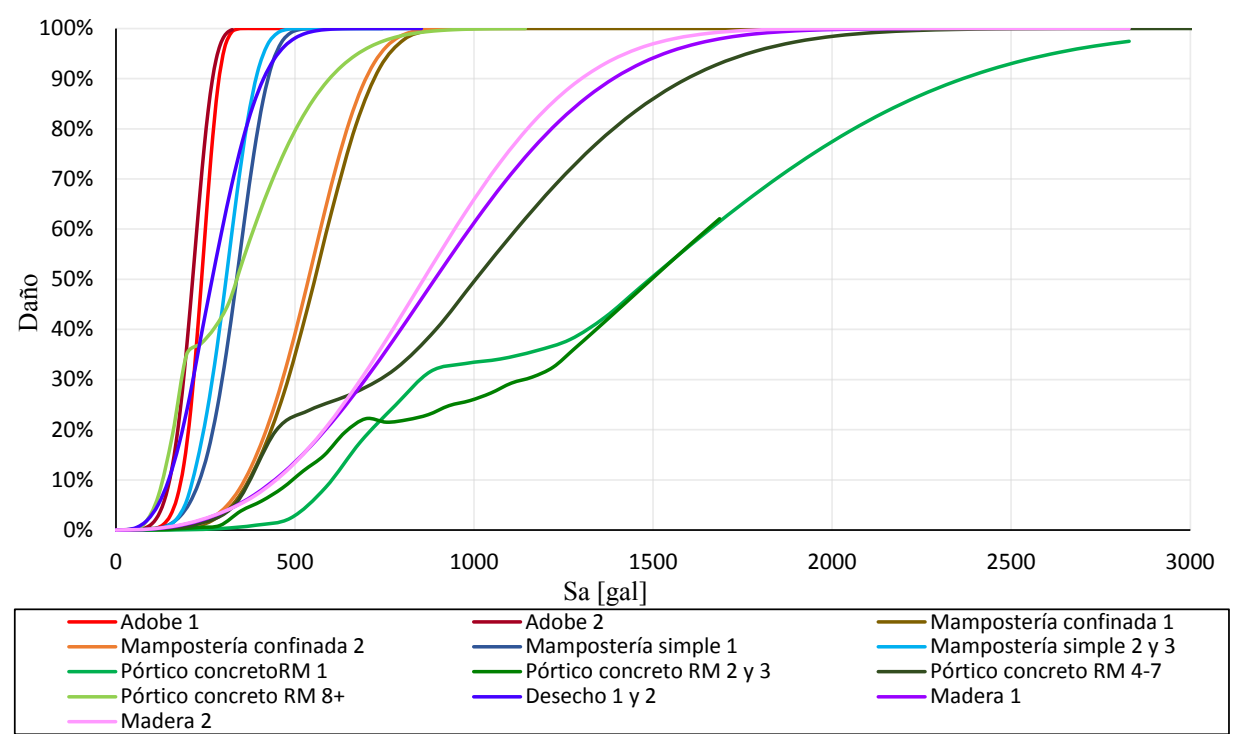

Figura 15. Curvas de vulnerabilidad para las edificaciones de Mendoza 


\section{Amenaza sísmica}

La amenaza sísmica a nivel de roca firme se evaluó haciendo uso del modelo anteriormente presentado en este artículo. Sin embargo, con el fin de caracterizar adecuadamente la amenaza a nivel urbano, es necesario incluir el efecto asociado a la respuesta dinámica de los suelos blandos de la ciudad. Con este fin, se incorporó al modelo la información reportada en el estudio de Microzonificación Sísmica de Mendoza (INPRES, 1989), el cual da cuenta de los efectos locales de sitio específicos de la ciudad.

En la Figura 16 se presentan mapas de amenaza uniforme (a nivel de roca firme) para la Gran Mendoza, en donde se pueden apreciar los cambios en la distribución de las aceleraciones máximas y espectrales, para diferentes periodos de retorno. Como puede observarse, el nivel de amenaza varía gradualmente del noroeste al sureste, en donde la esquina noroccidental tiene el mayor nivel de amenaza mientras que la esquina suroriental tiene el menor nivel de amenaza; esto se debe a la presencia de la fuente sismogénica Cerro La Cal - Divisadero Largo, la cual pasa por el noroeste de la ciudad y cuyo buzamiento es hacia el oeste.
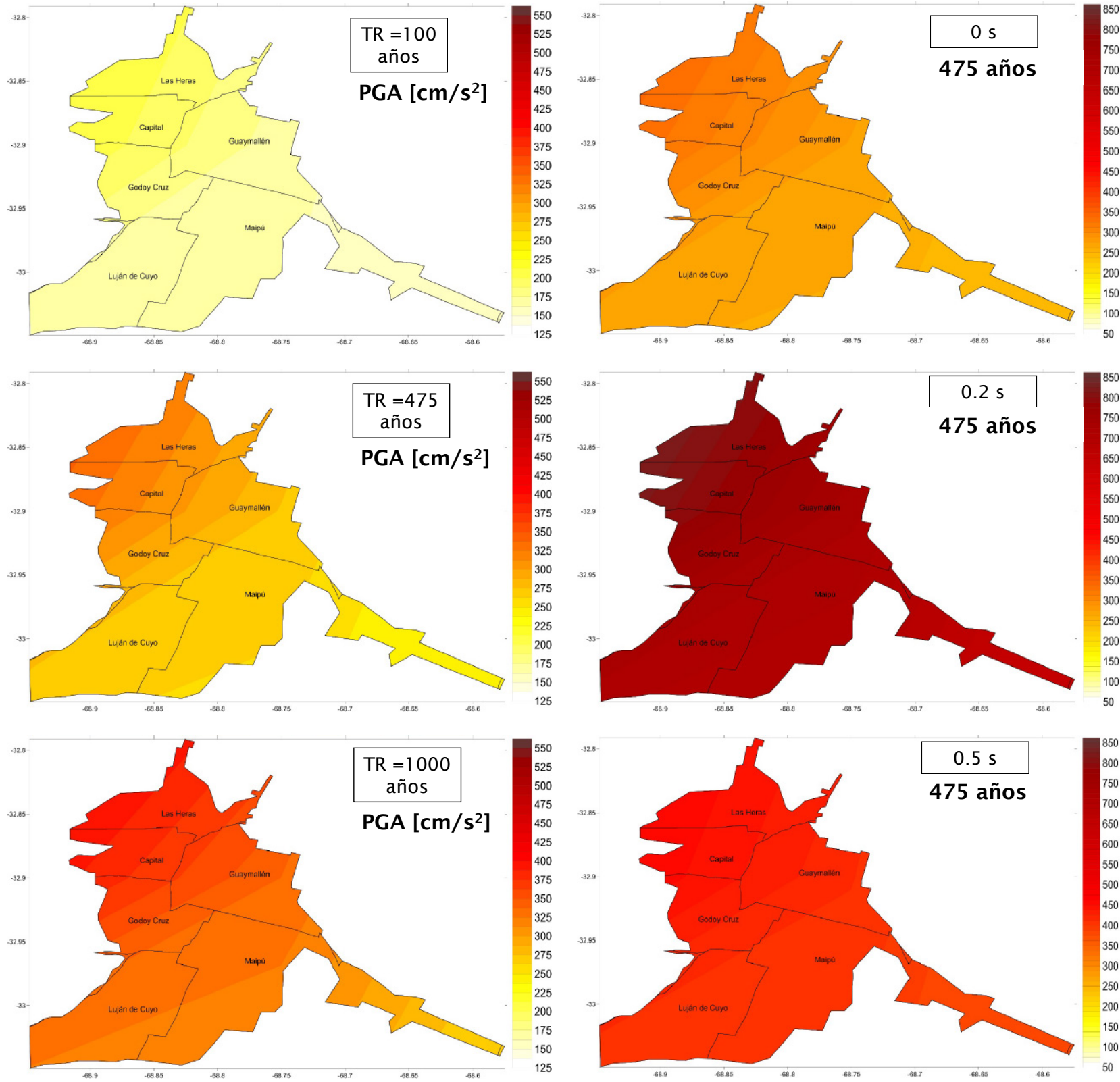

Figura 16. Mapas de amenaza en roca para PGA y varios periodos de retorno (izquierda) y para 475 años de periodo de retorno y varios periodos estructurales (derecho) 
A pesar de que en el marco del estudio de INPRES (1989) se realizaron numerosos sondeos y ensayos, la información no procesada de campo asociada a la investigación geotécnica del subsuelo no pudo ser obtenida. Por esta razón fue necesario incorporar los efectos de sitio de manera simplificada haciendo uso únicamente de los resultados existentes. En la Microzonificación Sísmica de Mendoza (INPRES, 1989) la ciudad se divide en dos cinco zonas, cada una caracterizada por un espectro de aceleraciones para diseño sismo resistente. La Figura 17 muestra la zonificación sísmica y los espectros de diseño para Mendoza.

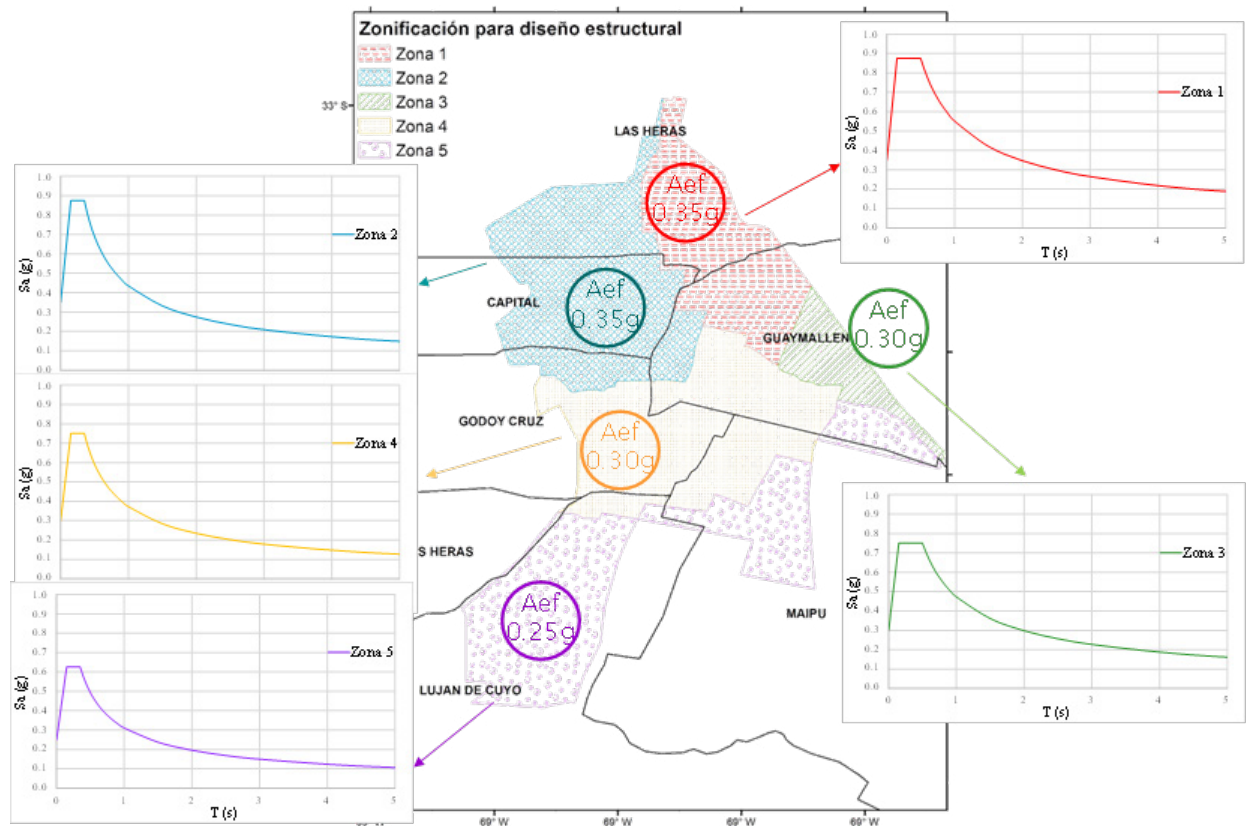

Figura 17. Mapa de la microzonificación sísmica para la Gran Mendoza (reproducido de INPRES, 1989)

Los espectros de diseño en superficie fueron usados como insumos para generar funciones de amplificación de las aceleraciones sísmicas. Las funciones de amplificación, como su nombre lo indica, entregan los factores de amplificación que deben aplicarse a las aceleraciones espectrales en roca firme, para transformarlas en aceleraciones espectrales a nivel de superficie del terreno. Para construir estas funciones, se empleó el siguiente procedimiento:

1. Dado que los espectros de diseño a nivel de superficie en INPRES (1989) están dados para un periodo de retorno de 200 años ( $10 \%$ de excedencia en 20 años), se selecciona el espectro de amenaza uniforme en roca, a partir del estudio de amenaza sísmica para toda la Argentina, para el mismo periodo de retorno. Estos espectros se muestran graficados simultáneamente en la Figura 18.

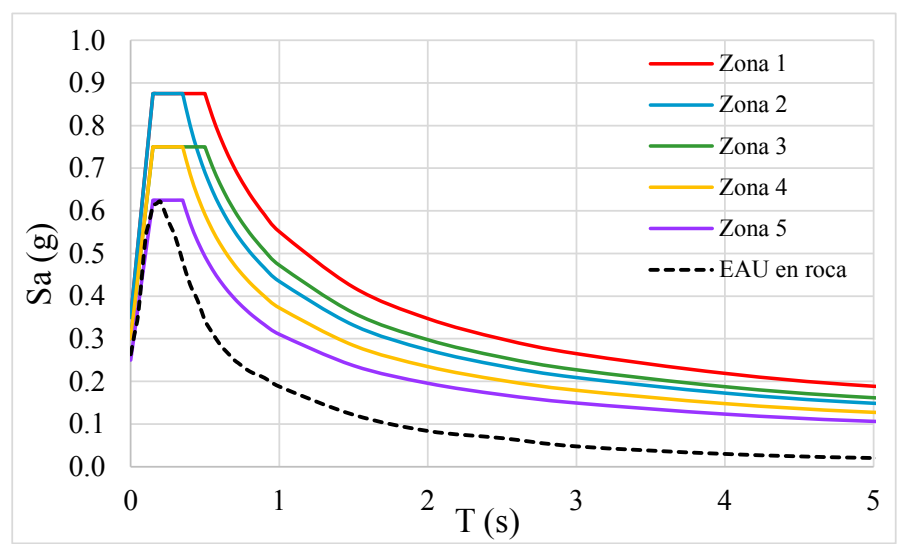

Figura 18. Espectros de diseño en suelo para las 5 zonas de la microzonificación y espectro de amenaza uniforme en roca. 
2. Dado que las funciones de amplificación representan la razón entre la aceleración en superficie y en roca, se realizó la división aritmética entre cada espectro de diseño en superficie y el de roca, como lo muestra la siguiente ecuación:

$$
F T_{\text {zonai }}=\frac{E D \text { en suelo }}{\text { zona } i}
$$

donde $F T$ es el valor de la función de amplificación o transferencia, ED es el espectro de diseño y $E A \cup$ es el espectro de amenaza uniforme.

Tras haber realizado esta conversión, finalmente se obtuvieron cinco funciones de transferencia para cada una de las cinco zonas de la microzonificación, la cuales se muestras en la Figura 19. Allí también se puede apreciar que, dado que la microzonificación contemplaba el área urbana de 1989, este límite fue modificado y ampliado para considerar el perímetro urbano actual. Estas funciones de transferencia son finalmente empleadas dentro del procedimiento de cálculo probabilista del riesgo para amplificar el movimiento fuerte generado por cada uno de los eventos sísmicos estocásticos que componen la amenaza sísmica.
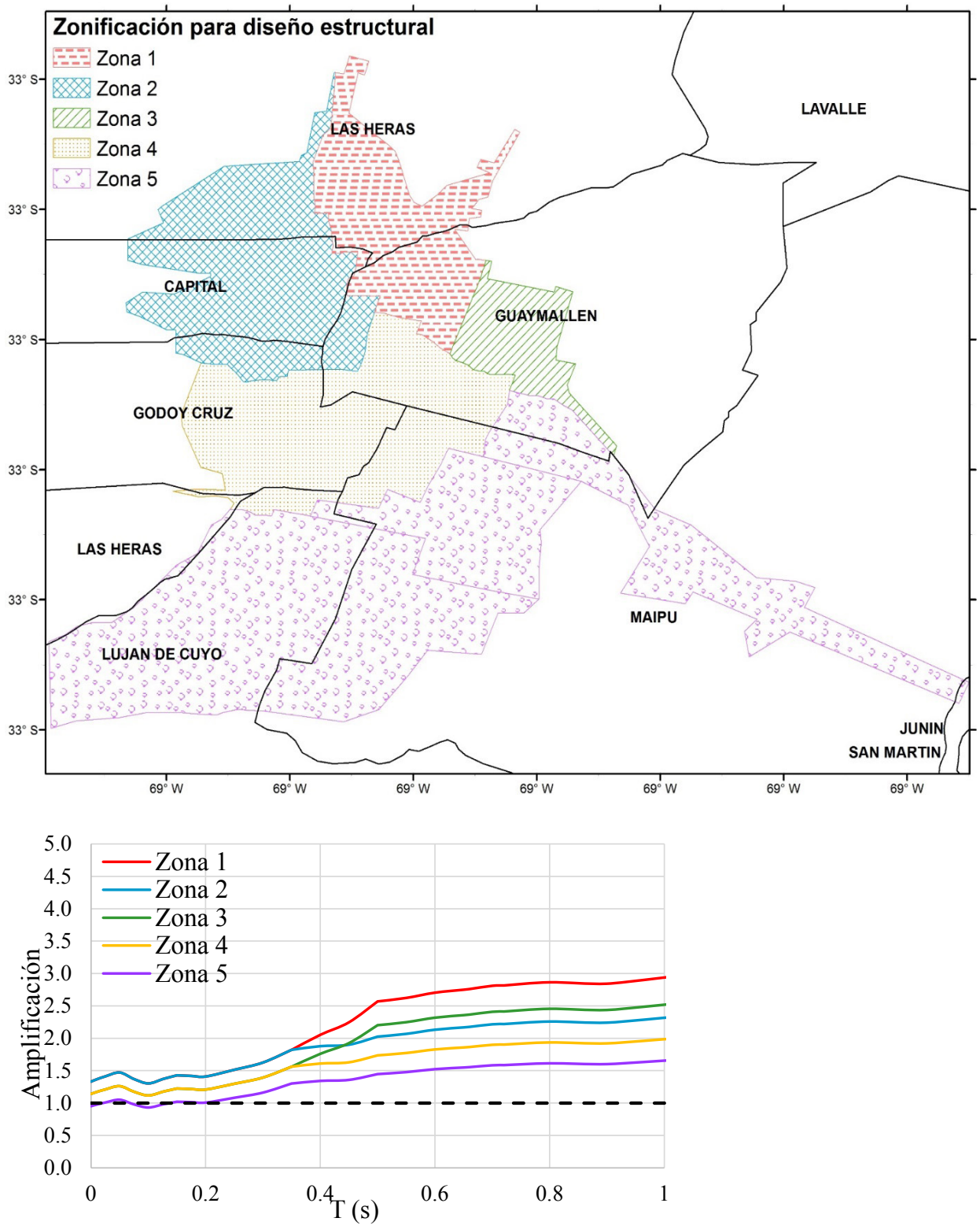

Figura 19. Microzonificación sísmica del Gran Mendoza (Arriba) y funciones de amplificación (abajo). 


\section{Evaluación probabilista de riesgo sísmico}

A continuación, se presentan los resultados de la evaluación de riesgo sísmico para el área de estudio; los resultados se presentan en términos de las mismas métricas presentadas en la evaluación a nivel nacional, la PAE y PML para diferentes períodos de retorno.

\begin{tabular}{|l|l|l|}
\hline \multicolumn{3}{|c|}{ Resultados } \\
\hline \multirow{2}{*}{ Valor expuesto } & ARS $\$ \times 10^{6}$ & 277,827 \\
\hline \multirow{2}{*}{ Pérdida anual esperada } & ARS $\$ \times 10^{6}$ & $1,637.917$ \\
\cline { 2 - 3 } & $\%$ oo & 5.895 \\
\hline \multicolumn{2}{|c|}{ PML } \\
\hline Periodo retorno & \multicolumn{2}{|c|}{ Pérdida } \\
\hline Años & ARS\$ $\$ 10^{6}$ & $\%$ \\
\hline 100 & $\$ 37,850.00$ & 13.62 \\
\hline 250 & $\$ 68,839.13$ & 24.78 \\
\hline 500 & $\$ 95,187.36$ & 34.26 \\
\hline 1000 & $\$ 119,643.76$ & 43.06 \\
\hline
\end{tabular}

Tabla 2. Resumen de resultados.

La pérdida anual esperada corresponde al 5.9 por mil con respecto al valor expuesto total del Gran Mendoza. Este nivel alto de riesgo sísmico, expresado a través de la pérdida anual esperada relativa, se puede explicar por características propias de la amenaza sísmica, los efectos de sitio y la vulnerabilidad física de los elementos en el Gran Mendoza. En primer lugar, y tal como se mostró en la amenaza sísmica, se cuenta en promedio con una aceleración cercana a $0.37 \mathrm{~g}$ en roca para la aceleración máxima del terreno y 475 años de período de retorno. Adicionalmente, considerando las funciones de transferencia propuestas de acuerdo a las zonas homogéneas de suelo, para ordenadas espectrales mayores a 0.3 segundos existen amplificaciones de entre 1.5 y hasta 3 veces, lo que, complementando con la vulnerabilidad de las edificaciones, principalmente aquellas de adobe y mampostería sin reforzamiento dan cuenta de estos resultados. Con respecto a las $\mathrm{PML}$, se presentan valores relativos entre el $14 \%$ para un periodo de retorno de 100 años, hasta el $43 \%$ para un periodo de retorno de 1000 años, los cuales confirman la condición de riesgo alto existente en Mendoza. La Figura 20 presenta las curvas de excedencia de pérdidas, PML y probabilidad de quiebra por riesgo sísmico para la ciudad de Mendoza.
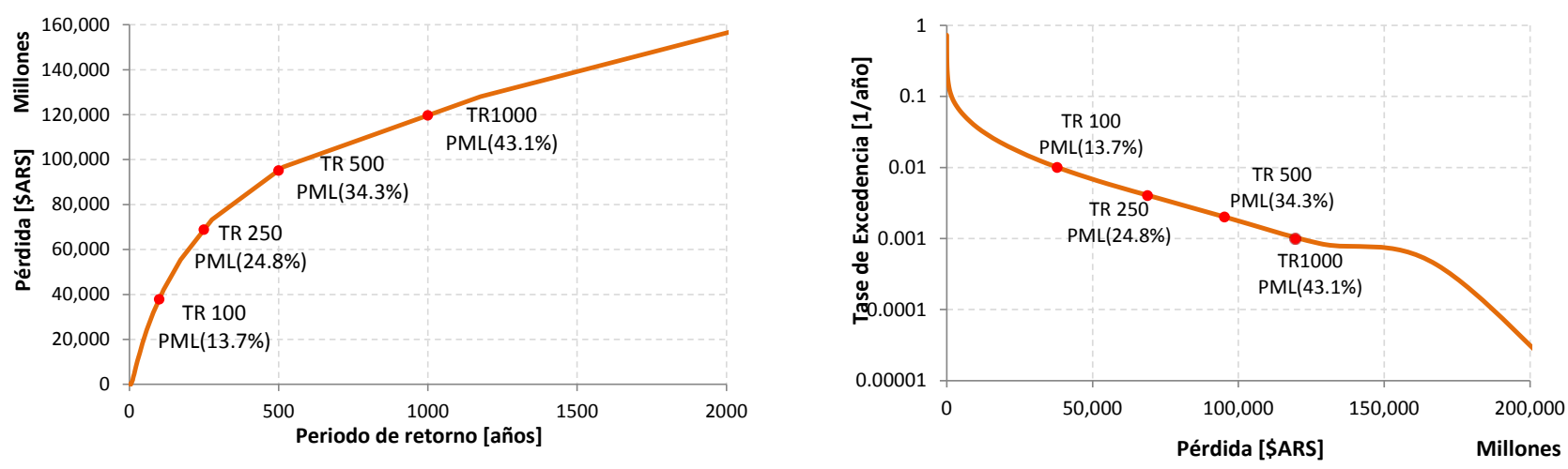

Figura 20. Curva de PML (arriba, izquierda), de excedencia de pérdidas (arriba, derecha) y probabilidad de quiebra (siguiente página). 


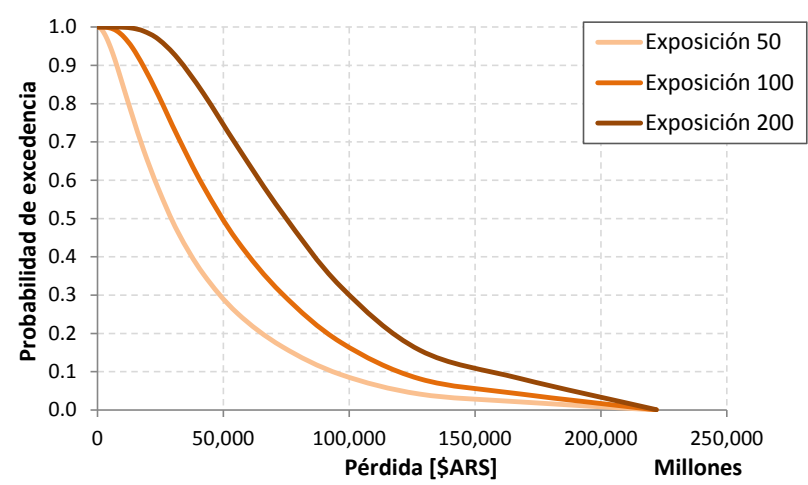

Figura 20. Curva de PML (página anterior, izquierda), de excedencia de pérdidas (página anterior, derecha) y probabilidad de quiebra (sobre estas líneas).

Finalmente, los resultados de riesgo también se pueden expresar en mapas que ilustran la distribución espacial de pérdidas. La Figura 21 y la Figura 22 presentan los mapas de riesgo sísmico en función de la pérdida anual esperada. El mapa de la Figura 21 se presenta en términos del valor monetario de la pérdida mientras que el de la Figura 22 se presenta en términos de dicho valor, normalizado por el valor expuesto de cada elemento, es decir, lo que se conoce como prima pura de riesgo, la cual es, para fines comparativos, la métrica más adecuada, dado que permite la mejor visualización de la distribución geográfica del riesgo.

De la Figura 21 se puede ver que las pérdidas más bajas se encuentran hacia las afueras del centro de la ciudad, es decir, los valores más altos están en el centro de la ciudad y estos van disminuyendo hacia la periferia. Esto se puede explicar porque es precisamente en el centro de la ciudad donde se concentra el mayor valor expuesto, causando que los valores de pérdida son más altos en este sector debido a que los valores expuestos también son altos. La Figura 22 muestra un cambio en el patrón de la Figura 21 ya que en este mapa las pérdidas están normalizadas con el valor expuesto de cada elemento (prima pura de riesgo), donde se puede ver que ahora las zonas en colores rojos se extienden un poco más hacia Las Heras y Godoy Cruz, del lado occidental de la ciudad; esto indica que son estas zonas las que tienen un mayor riesgo sísmico dado que son las que mayores pérdidas anuales esperadas presentan, con respecto a su valor de reposición.

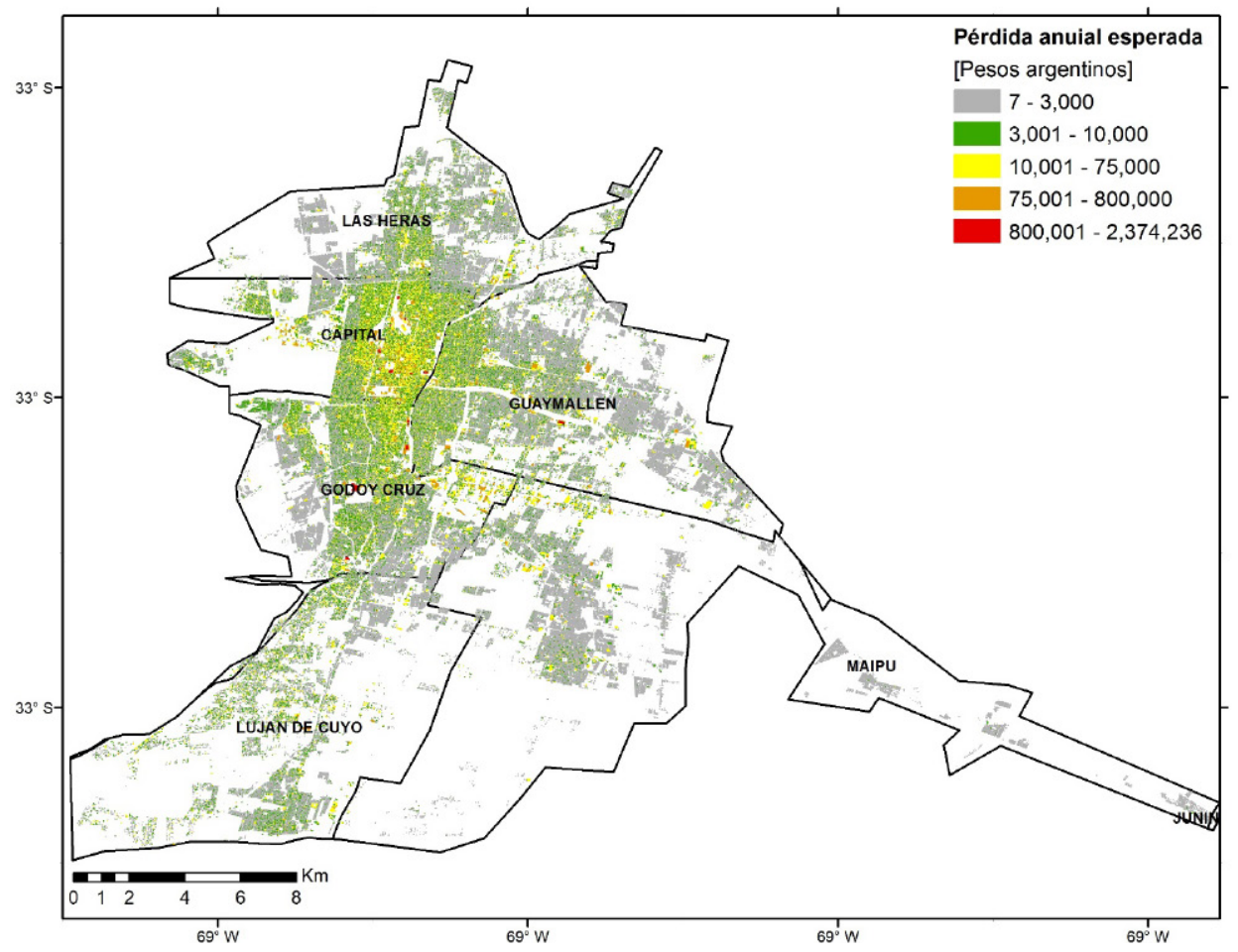

Figura 2l. Pérdida anual esperada absoluta. 


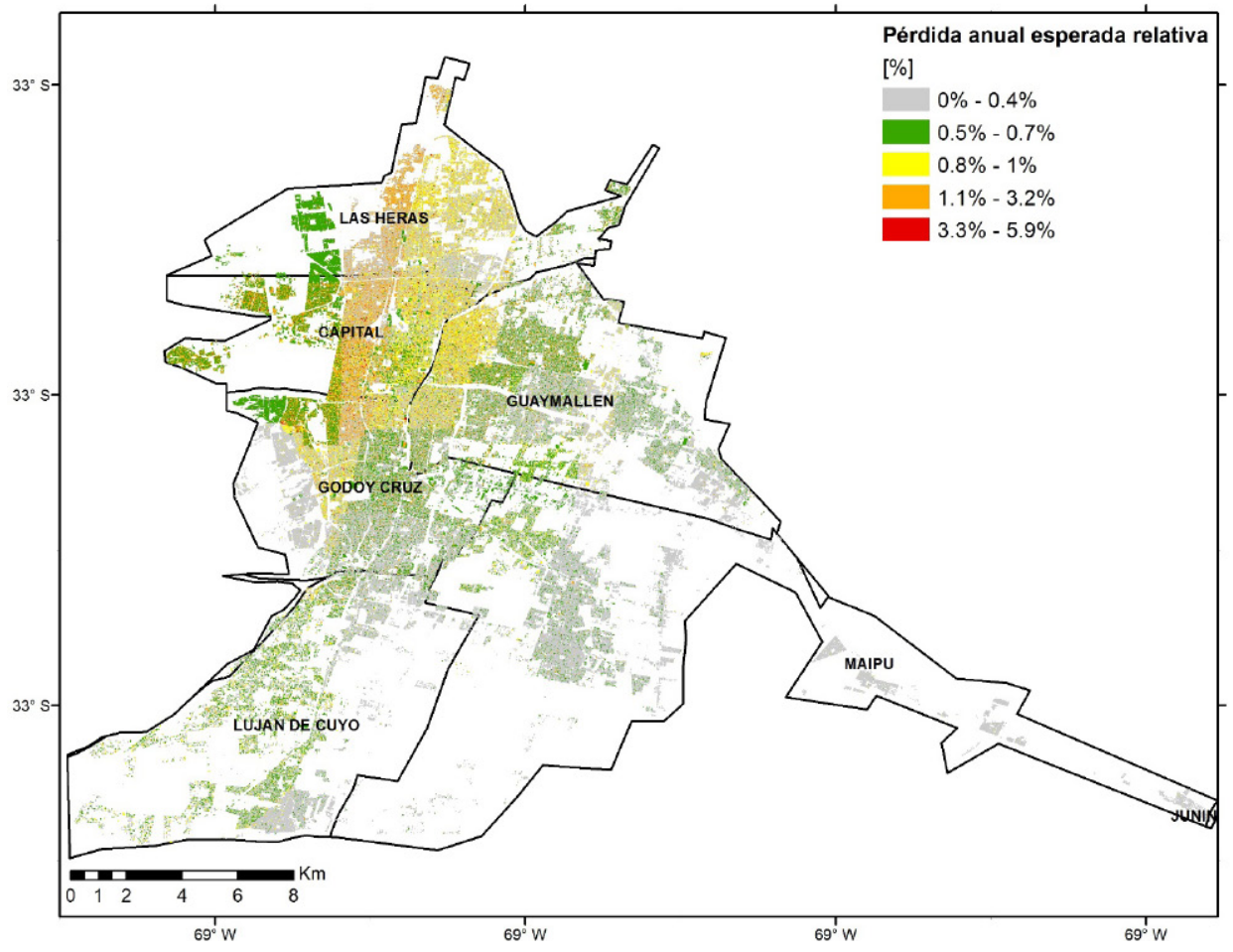

Figura 22. Pérdida anual esperada relativa al valor expuesto

\section{CONCLUSIONES}

La evaluación del riesgo de desastres permite conocer anticipadamente las cuantías de pérdidas asociadas a la ocurrencia natural de los terremotos, por medio de la integración en un modelo matemático de todos los aspectos relevantes en la generación de pérdidas económicas, de vidas y la afectación en general. La forma más adecuada de llevar a cabo evaluaciones de riesgo es mediante un enfoque probabilista, en el cual son involucradas racionalmente las incertidumbres en la definición y cálculo de la amenaza, la exposición y la vulnerabilidad. Las métricas de riesgo presentadas en este trabajo permiten dimensionar cifras y probabilidades de ocurrencia de pérdidas asociadas a terremoto, dentro de todo el portafolio de activos expuestos de Chile y Argentina, brindando así una medida cuantitativa del riesgo para facilitar el proceso de toma de decisiones.

La evaluación del riesgo sísmico presentada en este trabajo incluye un modelo de amenaza símica de última generación, el cual condensa todos los aspectos sismo-tectónicos relevantes en la región del cono sur, y aplica metodologías de punta en la evaluación de sismicidad, atenuación y amenaza en general. Los resultados obtenidos sólo desde la perspectiva de la amenaza, son útiles por si solos, por ejemplo, en la actualización de las normativas sismo resistentes nacionales o en la microzonificación sísmica de ciudades importantes, entre otros usos. La evaluación de amenaza constituye una base para el desarrollo de futuros proyectos de investigación asociados a la evaluación del riesgo, la modelación de otras amenazas (tsunami, deslizamientos), y la evaluación de la vulnerabilidad de construcciones e infraestructura crítica prioritaria.

Empleando la misma aritmética que para las evaluaciones nacionales, se calculó el riesgo sísmico de la ciudad de Mendoza, en Argentina. Para este tipo de evaluaciones locales se requiere de información mucho más detallada, como, por ejemplo, el catastro para la generación de una base de exposición a nivel de edificaciones, o la microzonificación sísmica de la ciudad. Esta es la primera vez que el riesgo sísmico de Mendoza es evaluado por medio de un enfoque totalmente probabilista, permitiendo así obtener las mismas métricas de riesgo que para el caso nacional, las cuales, por supuesto, deben ser interpretadas a la luz de las implicaciones específicamente para la ciudad. Estas evaluaciones son el primer insumo para formular estrategias integrales de gestión del riesgo sísmico, en donde se requiere tomar decisiones informadas, no sólo del nivel de afectación posible tras un terremoto, sino de la probabilidad que el evento se presente y las incertidumbres inherentes a este tipo de proceso tectónicos y los efectos que causan. 


\section{REFERENCIAS}

Armijo, R., Rauld, R., Thiele, R., Vargas, G., Campos, J., Lacassin, R. \& Kausel, E. (2010). The West Andean Thrust, the San Ramón Fault, and the seismic hazard for Santiago, Chile. Tectonics, 29.

Bernal, G.A. (2014). Metodología para la modelación, cálculo y calibración de parámetros de la amenaza sísmica para la evaluación probabilista del riesgo. Tesis doctoral. Barcelona, España: Universidad Politécnica de Cataluña.

Bernal, G.A. \& Cardona, O.D. (2015). Calibración de funciones de atenuación basadas en el espectro de fuente radiado y su aplicación en Colombia. Serie: Monografías de Ingeniería Sísmica. IS-71. Barcelona, España: CIMNE.

Cardona, O.D., Ordaz, M., Reinoso, E., Yamín, L.E. \& Barbat, A.H. (2012). CAPRA - Comprehensive Approach to Probabilistic Risk Assessment: International Initiative for Risk Management Effectiveness. Proceedings of the 15th World Conference on Earthquake Engineering. Lisbon, Portugal.

Carrapa, B. \& DeCelles, P.G. (2008). Eocene exhumation and basin development in the Puna of northwestern Argentina. Tectonics, 27.

Cornell, C.A. (1968). Engineering Seismic Risk Analysis. Bulletin of the Seismological Society of America, 58, 1583-16o6.

Costa, C. (2004). ¿Microtectónica en el Cuaternario?: Métodos y aplicaciones de la pelosismología. Revista de la Asociación Geológica Argentina Serie D Publicación Especial 7, 9-19.

Costa, C., Machette, M.N., Dart, R.L., Bastías, H.E., Paredes, JD., Perucca, L.P., Tello, G.E., Haller, K.M. (2000). Maps and database of quaternary faults and folds in Argentina. Open-file report oo-o1o8. USGS (United States Geological Service).

CSN (Centro Sismológico Nacional de la Universidad de Chile). (s.f). Sismicidad y terremotos en Chile. Recuperado el 1 de Abril de 2017, del sitio web de CSN: http://www.sismologia.cl/pdf/difusion/oo1_ terremotos_y_sismicidad_chile.pdf

DeCelles, P., Carrapa, B., Horton, B. \& Gehrels, G. (2011). Cenozioc foreland basin system in the central Andes of northwestern Argentina: Implications for Andean geodynamics and modes of deformation. Tectonics, 30 .

Deeken, A., Sobel, E. R., Coutand, I., Haschke, M., Riller, U. \& Strecker, M. (2006). Development of the sputhern Eastern Cordillera, NW Argnetina, constrained by apatite fission track thermochronology: From early Cretaceous extension to middle Miocene shortening. Tectonics, 25.

Esteva, L. (1967). Criterios para la construcción de espectros de diseño sísmico. Memorias de la zer Simposio Panamericano de Estructuras. Caracas, Venezuela.

Fazzito, S.Y. (2011). Estudios geofísicos aplicados a la neotectónica de la falla El Tigre, Precordillera de San Juan. Tesis Doctoral. Buenos Aires: Universidad de Buenos Aires.

Iaffa, D., Sabat, F., Muñoz, J.A. \& Carrera, N. (2013). Basin fragmentation controlled by tectonic inversion and basement uplift in Sierras Pampeanas and Santa Bárabara System, northwest Argentina. Londres: Geological Society London Special Publications. Recuperado el 1 de Abril de 2017, del sitio web de ResearchGate: https://www.researchgate.net/publication/236990132_Basin_fragmentation_ controlled_by_tectonic_inversion_and_basement_uplift_in_Sierras_Pampeanas_and_Santa_Brbara_ System_northwest_Argentina

INPRES (Instituto Nacional de Prevención Sísmica). (1989). Seismic microzonation of Gran Mendoza. Buenos Aires: INPRES.

ISC-GEM (International Seismologcal Centre-Global Earthquake Model Foundation). (2014). ISC-GEM Global instrumental earthquake catalogue V1.05 [Data set]. Recuperado el 1 de Abril de 2017, del sitio web de ISC-GEM: http://www.isc.ac.uk/iscgem/ 
Jordan, T. \& Allmendinger, R. (1986). The Sierras Pampeanas of Argentina: A modern analogue of rocky mountain foreland deformation. American Journal of Science, 286, 737-764.

Kendrick, E., Bevis, M., Smalley, R., Brooks, B., Vargas, R.B., Lauría, E. \& Fortes, L.P.S. (2003). The Nazca-South America Euler vector and its rate of change. Journal of South American Earth Sciences, 16, 125-131.

Kley, J. \& Monaldi, C. (1999). Estructura de las Sierras Subandinas y del Sistema de Santa Bárbara. En González-Bonorino, G., Omarini, R., Viramonte, J (Eds.), Geología del Noroeste Argentino (pp. 415-425). Salta, Argentina: Relatorio, XIV Congreso Geológico Argentino.

Lavenu, A., Thiele, R., Machette, M.N., Dart, R.L., Bradley, L. \& Haller, K.M. (200o). Maps and database of quaternary faults in Bolivia and Chile. Open-file report oo-283. USGS (United States Geological Service).

Leyton, F., Ruiz, S. \& Sepúlveda, S.A. (2010). Reevaluación del peligro sísmico probabilístico en Chile central. Andean Geology, 37 (2), 455-472.

Marulanda, M. (2013). Modelación probabilista de pérdidas económicas por sismo para la estimación de la vulnerabilidad fiscal del Estado y la gestión financiera del riesgo soberano. Tesis doctoral. Barcelona, España: Universitat Politecnica de Catalunya.

Masaferro, J. L., Bulnes, M., Poblet, J. \& Casson, N. (2003). Kinematic evolution and fracture prediction of the Valle Morado structure inferred from 3-D seismic data, Salta province, northwest Argentina. AAPG Bulletin, 87 (7), 1083-1104.

Núñez, I.A. (2014). Nuevo peligro sísmico para Chile. Tesis de pregrado. Santiago de Chile: Universidad de Chile.

Ordaz, M. (200o). Metodología para la evaluación del riesgo sísmico enfocada a la gerencia de seguros por terremoto. México D.F.: UNAM.

Ordaz, M., Martinelli, F., Aguilar, A., Arboleda, J., Meletti, C. \& D’Amico, V. (2014). CRISIS, Program for computing seismic hazard. México D.F.: Instituto de Ingeniería UNAM.

Perucca, L.P. \& Vargas, N. (2014). Neotectónica de la provincia de San Juan, centro-oeste de Argentina. Boletín de la Sociedad Geológica Mexicana, 66(2), 291-304.

Pingel, H., Strecker, M., Alonso, R. \& Schmitt, A. (2013). Neotectonic basin and landscape evolution in the Eastern Cordillera of NW Argentina, Humahuaca Basin ( $\left.24^{\circ} \mathrm{S}\right)$. Basin Research, 25, 554-573.

Ramos, V., Cristallini, E. \& Pérez, D. (2001). The Pampean flat-slab of the Central Andes. Journal of South American Earth Sciences, 15, 59-78.

Sadigh, K., Chang, C-Y., Egan, J.A., Makdisi, F. \& Youngs, R.R. (1997). Attenuation relationships for shallow crustal earthquakes based on California string motion data. Seismological Research Letter, $68,180-189$.

Salomon, E., Schimdt, S., Hetzel, R., Mingorance, F. \& Hampel, A. (2013). Repeated folding during late holocene earthquakes on the La Cal Thrust Fault near Mendoza City (Argentina). Bulletin of the Seismological Society of America, 103 (2A), 936-949.

SEGEMAR (Servicio Geológico Minero Argentino). (2015). [servicio WMS] Fallas Argentina Escala 1:2.500.ooo. Recuperado el 30 de Marzo de 2017, del sitio web de SEGEMAR: http://sig.segemar.gov.ar/

SEREMI (Secretaría Regional Ministerial Metropolitana de Vivienda y Urbanismo). (2011). Informe Etapa 2, Anexo I "Estimación del peligro sísmico" (Estudio "Riesgo y adecuación plan regulador comunal de San José de Maipo”, ID N640-6-LP11). Santiago de Chile: Ministerio de Vivienda y Urbanismo.

SERNAGEOMIN (Servicio Nacional de Geología y Minería). (2014). [Portal de mapas interactivos]. Geovisor Portal Geológico. Recuperado el 30 de Marzo de 2017, del sitio web de SERNAGEOMIN: http://geoportal.sernageomin.cl/geovisor/GeoVisor/index.html 
Silva, N.A. (2008). Caracterización y determinación del peligro sísmico en la Región Metropolitana. Tesis de pregrado. Santiago de Chile: Universidad de Chile.

Storchak, D. A., Di Giacomo, D., Bondár, I., Engdahl, E. R., Harris, J., Lee, W., Villaseñor, A., Bormann, P. (2013). Public Release of the ISC-GEM Global Instrumental Earthquake Catalogue (1900-2009). Seismological Research Letters, 84 (5), 810-815.

Tinti, S. \& Mulargia, F. (1985). An improved method for the analysis of the completeness of a seismic catalogue. Lettere Al Nuovo Cimento Series 2, 42(1), 21-27.

USGS (United States Geological Survey). (2013). Earthquake archive search [Data set]. Recuperado el 30 de Marzo de 2017, del sitio web de USGS: http://earthquake.usgs.gov/earthquakes/search/

Vargas, G., Rebolledo, S., Sepúlveda, S.A., Lahsen, A., Thiele, R., Townley, B., Padilla, C., Rauld, R., Herrera, M.J. \& Lara, M. (2012). Submarine earthquake ruptura, active faulting and volcanism along the major Liquiñe-Ofqui Fault Zone and implications for the seismic hazard assessment in the Patagonian Andes. Andean Geology, 40 (1), 141-171.

Zhao, J., Zhang, X., Asano, A., Ohno, Y., Oouchi, T., Takahashi, T., Ogawa, H., Irikura, K., Thio, H.K. \& Sommerville, P.G. (2006). Attenuation relations of strong ground motion in Japan using site classification based in predominant period. Bulletin of the Seismological Society of America, 898-913. 